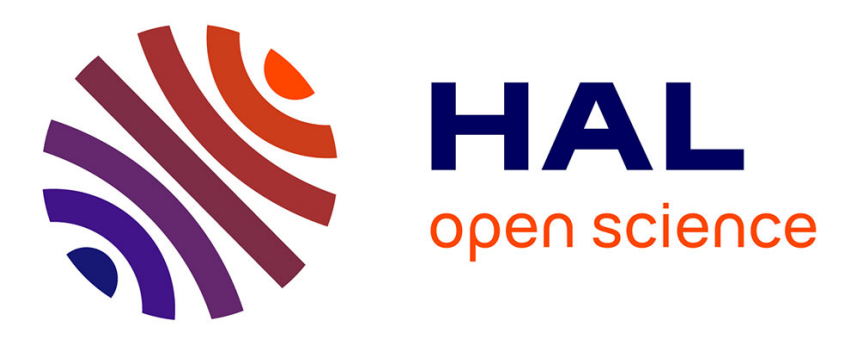

\title{
Association optimization based on access fairness for Wi-Fi networks
}

\author{
Mohammed Amer, Isabelle Guérin-Lassous, Anthony Busson
}

\section{To cite this version:}

Mohammed Amer, Isabelle Guérin-Lassous, Anthony Busson. Association optimization based on access fairness for Wi-Fi networks. Computer Networks, 2018, 137, pp.173 - 188. 10.1016/j.comnet.2018.03.004 . hal-01906319

\section{HAL Id: hal-01906319 https://hal.inria.fr/hal-01906319}

Submitted on 30 Oct 2018

HAL is a multi-disciplinary open access archive for the deposit and dissemination of scientific research documents, whether they are published or not. The documents may come from teaching and research institutions in France or abroad, or from public or private research centers.
L'archive ouverte pluridisciplinaire HAL, est destinée au dépôt et à la diffusion de documents scientifiques de niveau recherche, publiés ou non, émanant des établissements d'enseignement et de recherche français ou étrangers, des laboratoires publics ou privés. 


\title{
Association Optimization based on Access Fairness for Wi-Fi Networks
}

\author{
Mohammed Amer ${ }^{\mathrm{a}}$, Anthony Busson ${ }^{\mathrm{a}}$, Isabelle Guérin Lassous ${ }^{\mathrm{a}}$ \\ ${ }^{a}$ Univ Lyon, UCB Lyon 1, CNRS, ENS de Lyon, Inria, LIP UMR 5668, 46 Allée \\ d'Italie, 69364 Lyon, France
}

\begin{abstract}
Densification of Wi-Fi networks has led to the possibility for a wireless station to choose between several access points (APs), improving coverage, wireless link quality and mobility. But densification of APs may generate interference, contention and decrease the global throughput as these APs have to share a limited number of channels. The recent trend in which Wi-Fi networks are managed in a centralized way offers the opportunity to alleviate this problem through a global optimization of the resource usage. In particular, optimizing the association step between APs and stations can increase the overall throughput and fairness between stations. In this paper, we propose an original solution to this optimization problem based on two contributions. First, we propose a mathematical model to evaluate and forecast the throughput obtainable for each station for a given association. The best association is then defined as the one that maximizes a logarithmic utility function using the stations' throughputs predicted by the model. The use of a logarithmic utility function allows to achieve a good trade-off between overall throughput and fairness. A heuristic based on a local search algorithm is used to propose approximate solutions to this optimization problem. It relies on a suitable neighborhood structure between associations. This approach has the benefit to be tuned according to the CPU and time constraints of the WLAN controller. A comparison between different heuristic versions and the optimum solution shows that the proposed heuristic offers solutions very close to the optimum with a significant gain of time. We also evaluated our solution with the simulator NS-3 on a large set of scenarios and configurations. It empirically demonstrates that our proposal improves the overall throughput and the fairness of the network.
\end{abstract}

Keywords: IEEE 802.11, wireless, association optimization, throughput 


\section{Introduction}

Wireless access to the Internet has observed an important growth this last decades to become the most popular in terms of traffic [1]. In companies, campus, or at home, the preferred wireless technology to access to the Internet is Wi-Fi, based on the family of IEEE 802.11 standards [2]. This significant success is partly due to the facility to deploy such networks, the penetration of mobile devices (laptops, smartphones, and tablets), and the obvious convenience for the user to stay connected while being mobile. But, the capacity of the Wi-Fi technology is limited. There are two unlicensed frequency bands, UNII and ISM, with respectively 3 and $820 \mathrm{MHz}$-orthogonal channels. In order to use transmission rates in concordance with the increase of traffic demand, advanced transmission techniques and channels merge have been recently standardized (IEEE 802.11n [3] and 802.11ac [4]). The high transmission rates offered by these technologies rely also, in practice, on a densification of Access Points (APs) belonging to the same Wi-Fi network, i.e. with the same Extended Service Set ID (ESSID). Such a densification aims to ensure an efficient coverage of a Wi-Fi network and allows stations to have at least an AP in their close vicinity, which guarantees high transmission rates. This densification is also amplified by the increase of the number of different Wi-Fi networks (different ESSID or SSID), and a station may observe, in its radio range, a large number of Wi-Fi networks [5, 6]. But the increase of transmission rates and densification are not the only factor that can provide a capacity growth of the wireless access. Channel spatial reuse and transmission rates cannot increase unlimitedly and their usage must be consequently optimized. The recent trend of the Wi-Fi technology market offers a technological framework that allows such optimizations. Wi-Fi architectures have, for most of the products, a mode where a controller is in charge of a Wi-Fi network (consisting of a set of APs) in terms of configuration, management and optimization. This breaks the traditional approach where APs take all their decisions (association, channel selection, etc.) in an autonomous way. Even if most of the existing solutions are proprietary, different standards have been proposed to offer common protocols to support these centralized services, like for instance, CAPWAP [7] standardized by IETF, and IEEE 802.11v [8]. Furthermore, some propositions suggest to manage Wi-Fi networks through the SDN paradigm [9]. 
The design of wireless LANs applies to a large number of parameters as the number of APs and their location, the frequencies/channels to assign, the associations between wireless stations and APs, etc. Optimization models and algorithms aim to reduce costs and to improve performance through a better management of the available resources. Unfortunately, even with the development of computational technologies and parallel processing, many of these problems cannot be solved optimally in a reasonable computational time because of their internal nature or size. Consequently, the challenge is to design algorithms or heuristics offering a good trade-off between optimality and complexity. A more complete description of these problems, models, as well as the main optimization tools are provided in [10, 11, 12, 13].

In this paper, we focus on the optimization of the association between stations and APs. By default, a station associates to the AP with the best signal quality, generally the one with the highest Radio Signal Strength Indicator (RSSI). The distribution of users among APs of the same Wi-Fi network is then dependent on the geographical locations of users and radio environment (path-loss, fading/shadowing) and not on the number of stations already associated to each AP. It may lead to poor performance for users as they may be attached to the same AP whereas some other APs are idle [14. Instead, the controller can distribute stations among APs in a centralized manner to optimize a given objective function, like, for instance, maximizing the global throughput of the network. The centralized association problem has been already addressed in [14, 15, 16, 17, 18, 19]. Most of these optimization models assume a time-based fairness between the APs and the stations [15, 16, 17, 19], i.e. assume that the time is equally shared between stations and APs. This assumption requires to apply an appropriate scheduling on each AP that must take into account different parameters like the packet sizes and the physical transmission rates. In practice, APs use very simple scheduling policies like a FIFO scheduling and the DCF (Distributed Coordination Function) mode of IEEE 802.11 provides an accessbased share of the medium between APs/stations. Therefore, considering an access-based fairness model for the medium share seems more appropriate. Very few solutions, based on explicit optimization models, use such an access-based fairness scheme. This is the case in [14], but the solution goal is to minimize the maximal load on all the APs. In our work, we opt for a logarithmic utility function. Its optimization offers a good tradeoff between the overall network throughput and the fairness of user throughputs. Thus, our solution belongs to proportional fairness solution. Finally, contrary to most 
of the proportional fairness solutions based on optimization models (and all considering a time-based fairness share), we evaluate our solution not only with an optimization solver but also with a network simulator.

The contributions of this paper are the following:

- We propose a centralized optimization model for the association step that is based on a logarithmic utility function. In this model, we consider that an AP allocates, in average, the same number of accesses to the medium to each station associated with it, compared to the papers of the literature where the AP allocates the same amount of time to each station. Our approach is thus more realistic as it corresponds to the current implementation of APs and to the IEEE 802.11 DCF mode.

- A local search heuristic is proposed to solve this problem (being NPhard). This heuristic has the benefit to be tuned according to time and CPU constraints of the wireless local area network (WLAN) controller. A deeper analysis of the complexity of this algorithm is given and shown compatible with a real-time implementation.

- Our solution has been implemented on the network simulator ns-3. Results show that the global throughput is significantly increased compared to the default RSSI association, and leads to an improvement of fairness. A deeper analysis points out that, thanks to our optimization, stations are more homogeneously shared among access points, and individual throughput per station is improved for almost all the stations.

- Simulations have been performed for numerous scenarios, including different traffic types (TCP/UDP) and load (saturated/non-saturated), different distribution of packet sizes and orthogonal/non-orthogonal channels. We also evaluate an on-line version of the proposed algorithm where the optimization is performed regularly to take into account departures and arrivals of the stations.

This paper is an extended version of [20]. Compared to [20], this new version includes, in addition to a new Related Work section, a more detailed version of our local search algorithm, a comparison of the proposed heuristic with the optimum solution and several versions of our heuristic with different initial configurations along with the computing times, a comparison of our solution with time-based fairness solutions, and a more detailed performance 
evaluation part with new scenarios including realistic and variable packet sizes extracted from a real traffic trace, non saturated networks and TCP traffic. The paper is organized as follows. In Section 2 we present works related to the optimization of Wi-Fi association. Then, in Section 3 , we present our mathematical model of the optimization problem when orthogonal and non-orthogonal channels are used. In Section 3.2, the proposed approach to solve this model is described. A performance evaluation of our solution based on ns-3 simulations is carried out in Section 4. Our solution is compared to the time-based fairness approach in Section 5. We conclude in Section 6 .

\section{Related Work}

In a IEEE 802.11-based infrastructure network, a wireless station must be associated with one access point to be allowed to use the network. When several access points, belonging to the same network (named ESS - Extended Service Set), are available within its reception range, the wireless station must select one access point. Most of the current IEEE 802.11 wireless interfaces select the access point according to the Received Signal Strength Index (RSSI). Several papers, discussed in this section, claim that the use of the RSSI metric is not an efficient approach and have proposed different solutions for the association. In Section 2.1, we classify these papers according to different criteria. In Section 2.2, we describe these papers with more details.

\begin{tabular}{|c|c|c|c|c|c|c|c|c|c|c|c|c|}
\hline Paper & Cnt & Dst & On & Off & $\mathrm{AbF}$ & TbF & Obj F & Metric & \begin{tabular}{|l} 
Down \\
\end{tabular} & Up & Sim & Exp \\
\hline Our solution & $\mathrm{X}$ & & $\mathrm{X}$ & $\mathrm{X}$ & $\mathrm{X}$ & & $\log$ & & $\mathrm{X}$ & & $\mathrm{ns}-3$ & \\
\hline 14 & $\mathrm{X}$ & & $\mathrm{X}$ & $\mathrm{X}$ & $\mathrm{X}$ & & & Max-min & $\mathrm{X}$ & & $\mathrm{C}$ & \\
\hline 15 & $\mathrm{X}$ & & & $\mathrm{X}$ & & $\mathrm{X}$ & $\log$ & & $\mathrm{X}$ & & $\mathrm{X}$ & \\
\hline 16 & $\mathrm{X}$ & & & $\mathrm{X}$ & & $\mathrm{X}$ & $\log$ & & & & $\mathrm{X}$ & \\
\hline 21 & & $\mathrm{X}$ & $\mathrm{X}$ & & $\mathrm{X}$ & & & Throughput & $\mathrm{X}$ & $\mathrm{X}$ & & $\mathrm{X}$ \\
\hline 22 & & $\mathrm{X}$ & $\mathrm{X}$ & & $\mathrm{X}$ & & & Min load & $\mathrm{X}$ & & $\mathrm{ns}-2$ & \\
\hline 17 & $\mathrm{X}$ & $\mathrm{X}$ & $\mathrm{X}$ & $\mathrm{X}$ & & $\mathrm{X}$ & $\log$ & $\log$ & $\mathrm{X}$ & & OMNet++ & \\
\hline 23 & & $\mathrm{X}$ & $\mathrm{X}$ & & $\mathrm{X}$ & & Max-AP & & $\mathrm{X}$ & & $\mathrm{X}$ & \\
\hline 24 & & $\mathrm{X}$ & $\mathrm{X}$ & & & & Max-flow & & $\mathrm{X}$ & & $\mathrm{C}$ & \\
\hline 18 & $\mathrm{X}$ & & $\mathrm{X}$ & & \multicolumn{2}{|c|}{ QoS } & & $\begin{array}{l}\text { signal quality } \\
\text { loss rate }\end{array}$ & $\mathrm{X}$ & $\mathrm{X}$ & & $\mathrm{X}$ \\
\hline 25 & $\mathrm{X}$ & & & $\mathrm{X}$ & $\mathrm{X}$ & & Max-min & & $\mathrm{X}$ & $\mathrm{X}$ & $\mathrm{ns}-3$ & \\
\hline 19 & $\mathrm{X}$ & $\mathrm{X}$ & & $\mathrm{X}$ & & $\mathrm{X}$ & $\log$ & & $\mathrm{X}$ & & Python & \\
\hline 26 & $\mathrm{X}$ & & & $\mathrm{X}$ & & $\mathrm{X}$ & $\log$ & & $\mathrm{X}$ & & $\mathrm{X}$ & \\
\hline 27,28 & $\mathrm{X}$ & & & $\mathrm{X}$ & & & $\mathrm{X}$ & & $\mathrm{X}$ & $\mathrm{X}$ & Matlab & \\
\hline 29 & & $\mathrm{X}$ & & $\mathrm{X}$ & & & $\mathrm{X}$ & & & & $\mathrm{X}$ & \\
\hline
\end{tabular}

Table 1: Related work taxonomy with 6 criteria taken into account and described in Section 2.1

\subsection{Classification of the proposed solutions}

In Table 1, we present a taxonomy of these papers according to different criteria: 
- centralized or distributed (Cnt/Dst): the association decision can be done in a central or distributed way. If the association is distributed in many IEEE 802.11 products, since each station locally makes its association choice based on the RSSI metric, the solutions proposed to improve the association are mainly centralized, as shown in Table 1. However, there also exist proposals that aim to improve the association based on the RSSI value in a distributed way. Some papers also propose both approaches [17, 19.

- on-line / off-line (On/Off): in an on-line approach, the association is triggered upon a new event. With this approach, every time there is a change in the network (i.e. a station moves or leaves the network, the network conditions sensed or measured by the stations change), the association is reconsidered. On the other hand, in the off-line approach, the solution is periodically executed from each current association in the network.

- access-based / time-based Fairness (AbF/TbF): all these papers consider specific bandwidth sharing between users. This share has an impact on the throughput that can be obtained by each station and is part of the optimization model. Some solutions assume that the medium share is fair in time ( $\mathrm{TbF})$, meaning that each station has the same proportion of times in average. Solutions based on this assumption aim to improve network performance while ensuring fairness in terms of service time between stations on the same access point. It ensures that each station obtains a throughput proportional to its physical transmission rate used for transmissions between the AP and the station. Other solutions consider that the share is fair in the number of accesses to the channel $(\mathrm{AbF})$. This guarantees that all stations on the same access point receive the same throughput regardless of the used physical rate. One solution considers that the share of the radio medium between users is driven by QoS requirements (denoted by 'QoS'). When considering downlink traffic, the fairness (in time or in access) is provided by APs that apply an appropriate scheduling to send packets to the stations according to the targeted fairness model.

- objective function / use of a metric (Obj F/Metric): When the solution is based on an optimization model, the solution seeks to 
optimize an objective function. 'log' refers to proportional fairness objective function. In these solutions, the authors look for a proportional fair association by optimizing the sum of logs of the users' throughput. 'Max-AP' refers to minimizing the maximum AP utilization. 'Maxflow' corresponds to the maximum flow problem. With 'Max-min', the goal is to maximize the minimal throughput among all the stations. When the proposed solution is distributed, it does not rely on an explicit optimization model. It is rather based on one (or more) metrics that is used by each station to select its AP, like, for instance, the achieved throughput by each station, the least loaded AP, the signal quality ot the packet loss rate.

- downlink / uplink traffic (Down/Up): in most of the proposed solutions, only downlink traffic (i.e. traffic sent from APs to stations) is considered in the association process because it represents the majority of traffic flows. Other solutions make no assumption on traffic direction and consider downlink and uplink traffic.

- simulation / experimentation (Sim/Exp): most of the proposed solutions are evaluated by simulation, while a limited number of solutions are evaluated experimentally. In many approaches based on an optimization model, solutions are evaluated numerically by using a tool that solves optimization problems, such as CVX in [15], or CPLEX in [16]. In some papers, the designed heuristics are coded and evaluated in Python or in C. In all these papers, only the model/algorithm is implemented and evaluated. The performance evaluation does not consider the behavior of the algorithms in more realistic networking scenarios taking into account the 802.11 DCF principles: realistic radio environment, hidden terminals/APs, IP/TCP layers, etc. On the other hand, only few papers perform realistic simulations, as in [17] where the simulations are realized with the OMNetpp simulator, with the ns-2 simulator in [22] and the ns-3 simulator in [25]. In the very short list of papers describing real experimentations, we can mention the work of [21] that uses the NITOS wireless testbed [21] and the ones of [18, 25] with a homemade testbed.

\subsection{Description of the proposed solutions}

In this section, we provide a short description of the papers listed in Table 1 . 
In [14, the authors propose a solution to manage user-AP association by ensuring a max-min fair bandwidth allocation. This optimal max-min allocation is obtained through load-balancing techniques. The authors also extend their off-line approach to an on-line solution that computes the offline solution each time the time elapsed between two calls to the optimization algorithm is longer than a given time threshold or when the maximal load among the access points is greater than an allowed load.

The authors of [15] consider the association problem between users/subscribers of a same provider and their set-top boxes. Most APs in urban areas share the same upstream provider. The idea is then to offer a collaborative solution where a user may associate to any box. The association is solved at the common upstream provider to maximize the provider network throughput as well as user experience. The solution is centralized and formulated as a proportional fairness optimization problem.

The authors of [16] address the association problem in multi-rate wireless LANs. They consider an objective function that achieves network-wide proportional fairness. This function tries to maximize the total logarithmic utility function expressed in terms of bandwidth, and to provide equal channel occupancy time to each user.

The authors of [21] propose a distributed solution. In this solution, a new metric, used by each station, is based on uplink and downlink activities and takes into account possible hidden stations.

To achieve load balancing that guarantees fairness among the stations, the authors of [22] have proposed a distributed and self-stabilized association scheme in multi-rate WLANs. The proposed scheme gradually balances the AP load in a distributed manner. In this approach, stations associate to APs according to their load.

The authors of [17] propose an algorithm for the user-AP association to achieve time-based fairness in multi-rate wireless LANs. The problem is formulated as a non-linear programming with an objective function of maximizing the total user bandwidth utilities in the whole network. The authors also propose a distributed version of their algorithm, initially designed in a central way.

In [23], the authors design a distributed algorithm that consider the problem of optimizing associations in $60-\mathrm{GHz}$ wireless access networks. The objective, in their problem formulation, is to minimize the maximum AP utilization while ensuring balanced and fair resource allocation.

The authors in 24] have formulated the AP association as a max-flow 
problem to improve the overall throughput, fairness, load balancing and also resilience to client mobility. The proposed distributed model relies on shared local information from multiple APs.

In [18], the author proposes a centralized approach based on fuzzy logic for load balancing. In this solution, the stations change of access point based on the load of the access points, the signal strength received by each station and QoS parameters such as loss rate and required deadlines.

In [25], the authors propose an AP association that maximizes the minimum user throughput. The problem is subject to constraints on the user migration cost implying overhead in handshaking, authentication and data flow management.

In [19] an association control algorithm is proposed to optimize the throughput in wireless LANs. The solution achieves load balancing between APs while considering users traffic demand.

In [26], the association optimization is formulated as a proportional fairness problem. It is solved periodically by a central controller. The solution considers the cost of handovers and the minimum throughput requirements (e.g. video or best-effort traffic) of each user when assigning users to APs.

The authors of [27, 28] propose a model to optimally design green wireless LANs. It consists in minimizing the power consumption of a WLAN, when the load is scarce, by powering-on a subset of APs and associating stations to them. The model takes into account data rates between stations and APs, users' mobility, and channel conditions.

To understand and improve the performance of several association control schemes, a theoretical framework that analyzes association problems in vehicular Wi-Fi networks is described in [29]. They formulate the association problem as a non-linear integer program taking into account influence of vehicles' mobility, available effective bit rate from APs, and handover cost. Their offline algorithm is compared to existing online algorithms.

\subsection{Contributions}

In this paper, we are interested in centralized association solutions based on explicit optimization models. Most of the optimization models, proposed for this problem, assume a time-based fairness between the APs and the stations [15, 16, 17, 19]. This approach does not correspond to most of the APs implementation which use FIFO queues rather than complex scheduling (necessary for time share). Moreover, to obtain a medium share in time in 
the whole network, the IEEE 802.11 DCF mode must be modified. Since the DCF mode of IEEE 802.11 provides an access-based share of the medium, an access-based fairness model for the medium share seems more appropriate. Very few solutions based on explicit optimization models use such an accessbased fairness scheme. This is the case in [14, 25], but the solution goal is to minimize the maximal load on all the APs. In this work, we maximize the logarithmic utility function. Finally, contrary to most of the proportional fairness solutions based on optimization models (and all considering a time-based fairness medium share), we evaluate our solution with a network simulator.

\section{Association Optimization}

\subsection{Problem Formulation}

In this section, we provide the model and the notations used for the mathematical formulation of our centralized solution. We consider a general 802.11 WLAN consisting of $m$ access points and $n$ wireless stations as illustrated in Figure 1. The set of APs is assumed to belong to the same extended service set (ESS) and is managed by a WLAN controller. The controller is in charge of determining the association. When a new station connects to the ESS, it first associates with the default AP which is, for most of the implementation, the one with the best RSSI. The controller can, according to our algorithm, change associations at regular intervals or when a particular event occurs (arrival/departure of stations for instance). We assume that the controller collects periodically the following measurements from APs:

- the current association,

- the conflicts between APs on each channel and co-channel,

- the data rates between APs and the stations.

It is worth noting that most of these measurements are already available on most of the AP products (e.g., Cisco Aironet Series APs). When the controller finds out a better association, it triggers the corresponding changes: through control frames, stations can be disassociated from the current AP and associated to the new one.

In this paper, we take into account only downlink traffic, from the APs to the stations, as downlink traffic is preponderant compared to uplink traffic. 


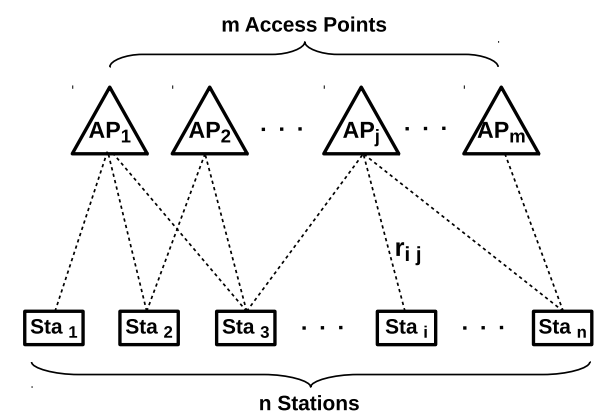

Figure 1: Access points and wireless stations in the network. Dotted lines represent the possible associations between APs and stations.

The amount of uplink traffic is considered negligible, or at least not significant, with regard to the downlink traffic [30, 31]. We also assume that the amount of data intended to the stations associated to the same AP is equal in average, or in a long term period. To this end, we assume that the mean number of frames transmitted to each station and the mean frame size are the same for each station. Obviously, it will not correspond to reality, but it allows to express the problem with an equal priority to each station. This assumption is motivated by different reasons: i) the optimization problem is thus addressed without privileging a station because it has more traffic than the others at a given time; ii) Internet traffic is quite sporadic and the time scale in terms of dynamics is very likely smaller than the one of the association problem, which implies that, in average, stations may receive the same amount of data; iii) the association problem output consists in associating stations with APs and the goal is not to directly set/reserve any resource for each station; consequently, stations that receive more traffic still benefit of the statistical multiplexing offered by the Wi-Fi technology.

We model the IEEE 802.11 infrastructure based wireless network through the following steps. We consider only the IEEE 802.11 DCF mode 32. Table 2 summarizes the different notations used throughout this paper.

The objective function that we optimize is based on the mean throughputs between APs and stations, denoted $d_{i j}(i \in\{1, \ldots, n\}$ and $j \in\{1, \ldots, m\})$. By convention, we set $d_{i j}=0$ if $S t a_{i}$ is not associated to $A P_{j}$. This throughput depends, among others, on the number of stations associated with the AP, and the corresponding link capacity. The link capacity $r_{i j}$ is defined here as the maximum amount of data that can be exchanged between $A P_{j}$ and $S t a_{i}$ 


\begin{tabular}{|c|l|}
\hline Symbol & Description \\
\hline \hline$m$ & Number of access points in the network \\
$n$ & Number of wireless stations in the network \\
$r_{i j}$ & Link capacity between $A P_{j}$ and $S t a_{i}$ \\
$t_{i j}$ & Mean transmission time of one frame from $A P_{j}$ to $S t a_{i}$ \\
$p_{i}$ & Mean frame size to be transmitted to $S t a_{i}$ \\
$d_{i j}$ & Mean throughput obtained by $S t a_{i}$ when associated to $A P_{j}$ \\
$D_{j}$ & Mean outgoing throughput of $A P_{j}$ \\
$L_{i j}$ & Mean number of frames transmitted from $A P_{j}$ to $S t a_{i}$ \\
$x_{i j}$ & 1 if $S t a_{i}$ is associated to $A P_{j}, 0$ otherwise \\
$s_{i j}$ & 1 if $A P_{i}$ is in sensing range of $A P_{j}, 0$ otherwise \\
\hline
\end{tabular}

Table 2: Notations

in one second. The throughput $d_{i j}$ is the throughput when considering the other stations and, in one of the proposed models (see Section 3.1.2), the other interfering APs. In other words, $d_{i j}$ takes into account the fact that the medium is shared whereas $r_{i j}$ does not.

We present our optimization problem under two variants. The first approach assumes that the channels used by the access points are orthogonal, meaning that they can not detect each other and can transmit at the same time without interfering. It is equivalent to assume that there are as many orthogonal channels as APs. Then, in the second approach, we consider that the number of orthogonal channels is limited. Consequently, APs which use the same channel and which are in the sensing range of each other share the medium. The formula that characterizes the throughput between an AP and a station is refined accordingly.

\subsubsection{Orthogonal channels}

We assume that all APs use different orthogonal channels, or equivalently the APs using the same channel are far enough to avoid any interference and signal detection. Therefore, each AP can be considered as an independent sub-network and the mean aggregate throughput for the whole Wi-Fi network is the sum of the mean AP throughputs. We begin by computing the mean overall throughput offered by an AP from which we derive the mean throughput between this AP and one of its associated stations.

The mean throughput $D_{j}$ of $A P_{j}$ is defined as the downlink throughput 
sent by this AP to the set of its associated stations:

$$
D_{j}=\sum_{i=1}^{n} d_{i j}
$$

It can also be expressed as the ratio between the mean quantity of data transmitted to all wireless stations associated to it and the time required for these transmissions:

$$
D_{j}=\frac{\sum_{i=1}^{n} L_{i j} p_{i} x_{i j}}{\sum_{i=1}^{n} L_{i j} t_{i j} x_{i j}}
$$

where $L_{i j}$ is the mean number of frames sent from $A P_{j}$ to $S t a_{i}, p_{i}$ is the mean size of these frames, $x_{i j}$ indicates if $S t a_{i}$ is associated to $A P_{j}$ (it is equal to 1 if it is true, and 0 otherwise) and $t_{i j}$ is the mean time to send a frame from $A P_{j}$ to $S t a_{i}$. This time is given by the ratio between the mean frame size and the link capacity:

$$
t_{i j}=\frac{p_{i}}{r_{i j}}
$$

By substituting (2) in (1), we get:

$$
D_{j}=\frac{\sum_{i=1}^{n} L_{i j} p_{i} x_{i j}}{\sum_{i=1}^{n} L_{i j} \frac{p_{i}}{r_{i j}} x_{i j}}
$$

As we assume that the mean number of frames transmitted to each station and the mean frame size are identical for each station, the mean overall throughput of an AP is then given by:

$$
D_{j}=\frac{\sum_{i=1}^{n} x_{i j}}{\sum_{i=1}^{n} \frac{x_{i j}}{r_{i j}}}
$$

Also, as we assume that the stations associated to the same AP receive the same amount of data in average, then the throughput of the AP is equally 
shared among its wireless stations. Therefore, the mean throughput $d_{i j}$ obtained by $S t a_{i}$ from its $A P_{j}$ becomes:

$$
d_{i j}=\frac{D_{j}}{\sum_{k=1}^{n} x_{k j}}
$$

Substituting $D_{j}$ in (5), we get:

$$
d_{i j}=\frac{1}{\sum_{k=1}^{n} \frac{x_{k j}}{r_{k j}}}
$$

From Equation (6), we can easily see that the mean throughput $d_{i j}$ of $S t a_{i}$ associated to $A P_{j}$ is the same for all stations associated to this AP, whereas they may experience different link capacities with this AP.

Our optimization aims to maximize the total downlink throughput for the whole network while ensuring fairness between wireless stations. In order to introduce fairness in the objective function, we use the logarithmic utility function proposed by Kelly in [33]. The association optimization problem with orthogonal channels can then be formulated as follows:

$$
\begin{array}{rll}
\max & \sum_{i=1}^{n} \log \left(\sum_{j=1}^{m} d_{i j} x_{i j}\right) & \\
\text { with } & d_{i j}=\frac{1}{\sum_{k=1}^{n} \frac{x_{k j}}{r_{k j}}} & 1 \leq i \leq n, 1 \leq j \leq m \\
\text { subject to } & \sum_{j=1}^{m} x_{i j}=1 & 1 \leq i \leq n, \\
& x_{i j} \in\{0,1\} & 1 \leq i \leq n, 1 \leq j \leq m, \\
& \text { if } r_{i j}=0 \text { then } x_{i j}=0 & 1 \leq i \leq n, 1 \leq j \leq m .
\end{array}
$$

The objective is thus to find the set of association variables $x_{i j}$ that maximizes the total network throughput while ensuring a certain fairness. The two first constraints are related to the association variables $x_{i j}$ and ensure that a station is connected to a single AP. The third constraint aims to guarantee that a wireless station cannot associate with an AP that is not within its receiving range. 


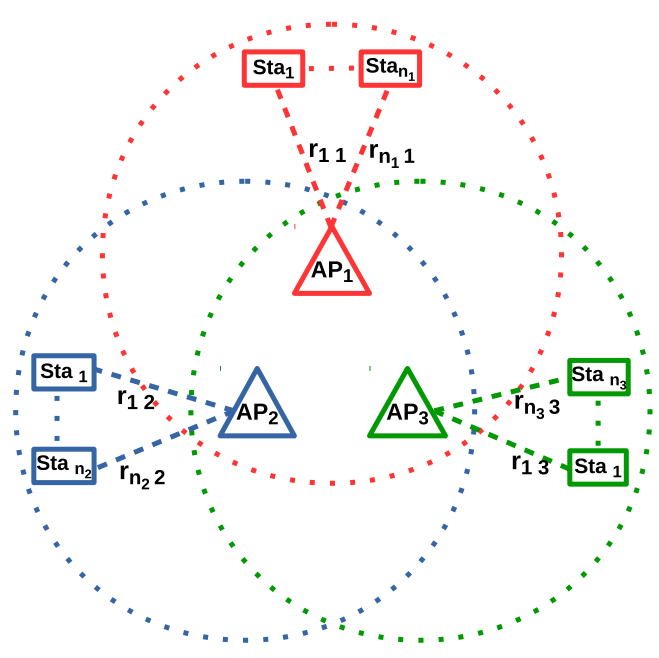

(a) Simple representation

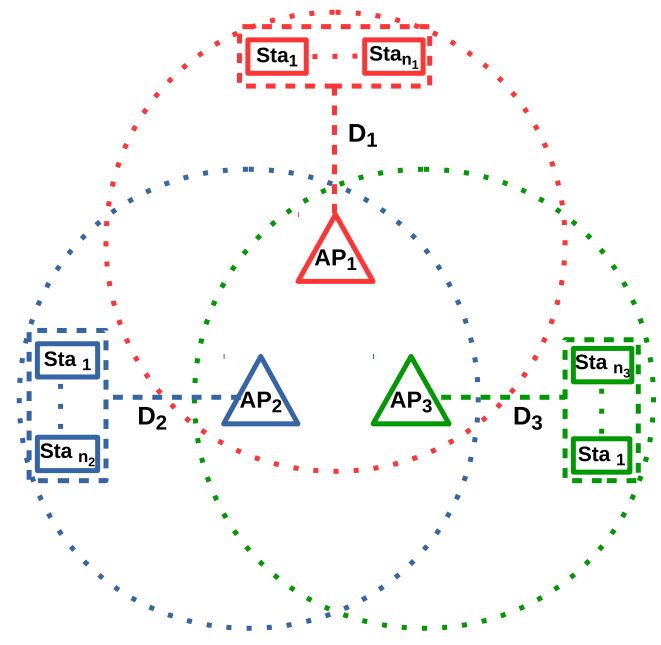

(b) Aggregated representation

Figure 2: Wireless network with non-orthogonal channels

\subsubsection{Non-orthogonal channels}

We propose to refine the model by considering non-orthogonal channels. A certain number of orthogonal channels are available but their number is limited, so several APs may use the same channel.

In practice, it is difficult to know the various interference that can undergo a radio signal in a wireless network. A source of interference can belong to the same Wi-Fi network, e.g. a nearby AP with the same SSID, part of the same Extended Service Set (ESS), or can be external such as another wireless network, or any radio source in the same frequency band. As information on interference and traffic are not easily available and unpredictable for external sources, we consider only interference that exist between APs of the same ESS.

We assume that the assignment of the channels to the APs has been set. Two APs will detect transmissions of each other if they use the same channel and are in the carrier sense range of each other. It leads to a share of the medium, as transmissions can not take place at the same time or collisions may happen if they transmit at the same time. The two APs are then in conflict.

According to the IEEE 802.11 DCF mode, APs in mutual conflict have equivalent opportunities to access the medium [34]. Therefore, we assume that the number of accesses to the medium is equal, in average, between 
conflicting APs. In Figure 2a, we represent three APs in mutual conflict.

To compute the mean throughput $D_{j}^{*}$ of $A P_{j}$ in presence of conflicts, we use Equation (6) and adapt it to this context. The throughput of stations associated to the same AP is seen as an aggregation, as shown in Figure 2b. $d_{i j}$ is then replaced by $D_{j}^{*}$. $D_{j}$ corresponds to the mean throughput of $A P_{j}$ without conflict, therefore $r_{k j}$ (which is the bandwidth obtained by one station without conflict) is replaced by $D_{j}$. Finally, in this context, the share comes from the APs in conflict: $x_{k j}$ is replaced by $s_{k j}$ that represents the number of APs in conflict with $A P_{j}$. Note that this adaptation of Equation (6) is possible because the opportunity to access the channel is the same for all APs in mutual conflicts (as the throughput of an AP is equally shared among its associated stations in the previous model):

$$
D_{j}^{*}=\frac{1}{\sum_{k=1}^{m} \frac{s_{k j}}{D_{k}}}
$$

Substituting (4) in (8), we get :

$$
D_{j}^{*}=\frac{1}{\sum_{k=1}^{m}\left(\frac{s_{k j}}{\sum_{i=1}^{n} x_{i k}} \sum_{i=1}^{n} \frac{x_{i k}}{r_{i k}}\right)}
$$

As for the case with orthogonal channels, we assume that the AP throughput is equally shared among the stations associated with it. Therefore, the mean throughput for a particular station is:

$$
d_{i j}^{*}=\frac{1}{\sum_{i^{\prime}=1}^{n} x_{i^{\prime} j}} \cdot \frac{1}{\sum_{k=1}^{m}\left(\frac{s_{k j}}{\sum_{i^{\prime}=1}^{n} x_{i^{\prime} k}} \cdot \sum_{i^{\prime}=1}^{n} \frac{x_{i^{\prime} k}}{r_{i^{\prime} k}}\right)}
$$

The formulation of the association optimization problem in a wireless network with non-orthogonal channels is given as follows: 


$$
\begin{aligned}
& \max \sum_{i=1}^{n} \log \left(\sum_{j=1}^{m} d_{i j} x_{i j}\right) \\
& \text { with } \quad d_{i j}=\frac{1}{\sum_{i^{\prime}=1}^{n} x_{i^{\prime} j}} \cdot \frac{1}{\sum_{k=1}^{m}\left(\frac{s_{k j}}{\sum_{i^{\prime}=1}^{n} x_{i^{\prime} k}} \cdot \sum_{i^{\prime}=1}^{n} \frac{x_{i^{\prime} k}}{r_{i^{\prime} k}}\right)} \\
& \text { subject to } \sum_{j=1}^{m} x_{i j}=1 \quad 1 \leq i \leq n \text {, } \\
& x_{i j} \in\{0,1\} \quad 1 \leq i \leq n, 1 \leq j \leq m, \\
& \text { if } r_{i j}=0 \text { then } x_{i j}=0 \quad 1 \leq i \leq n, 1 \leq j \leq m \text {. }
\end{aligned}
$$

The objective here is to maximize the overall network throughput while ensuring a certain fairness between the wireless stations when the APs use non-orthogonal channels. The expression of the mean throughput between an AP and an associated station has changed, compared to the orthogonal channel case, to take into account conflicts between APs. The constraints are the same as in the orthogonal channel case.

\subsection{Optimization problem solving}

The optimization association problem is formulated, in the previous section, as a centralized optimization approach based on the use of a logarithmic utility function. The problem is modeled as a non-linear programming problem with binary decision variables representing the association of wireless stations to APs, which is known to be NP-Hard [35].

Most of the studies that deal with optimization of Wi-Fi associations, and that have been presented in Section 2 [15, 17, 16, 19], use approximation algorithms based on relaxation to a non-linear convex program. It allows them to apply the rounding process proposed by Shmoys and Tardos for the generalized assignment problem [36], to provide binary values of the association variable $x_{i j}$. This often does not allow an exact solution of the problem in a reasonable computational time.

Instead, to solve our optimization problem, we propose an iterative heuristic based on the principle of local search, also called descent or iterative improvement. Local search is an important class of heuristics used to solve combinatorial optimization problems. The key idea of a local search algorithm is to start from an initial feasible solution and iteratively find, at each 
iteration, a solution called a best neighbor that improves the objective function [37]. The main benefits of local search lie in its simplicity and its iterative process which can stop the optimization process at any time to comply with a constraint like the computation time for instance. In contrast to constructive approaches, local search algorithms consider only complete feasible solutions during the search. The proposed algorithm has then the advantage to improve Wi-Fi associations at each iteration, and can be stopped at any time with a feasible solution. The time that the system spends in computing a solution can thus be bounded and tuned.

Our iterative local search method is based on two essential elements: a neighborhood structure and a procedure exploiting this neighborhood. The method can be summarized as follows:

1. It starts with an initial feasible solution.

2. At each iteration, it chooses, among all the neighbors of the current solution, one of the solutions that maximizes the objective function. This neighbor becomes the current solution on which to apply the next iteration.

We present in the two next paragraphs, the notion of neighborhood, and a more detailed version of the local search algorithm.

\subsubsection{Neighborhood structure}

Let $X\left(X=\left(x_{i j}\right)_{1 \leq i \leq n, 1 \leq j \leq m}\right)$ be a feasible solution of the optimization problem. $X$ is a $n \times m$ matrix (we remind that $n$ is the number of stations and $m$ the number of APs). Its terms $x_{i, j}$ correspond to the association variables of the optimization problem.

$$
X=\left(\begin{array}{ccccccc}
x_{1,1} & \cdots & x_{1, j} & \cdots & x_{1, j \prime} & \cdots & x_{1, m} \\
x_{2,1} & \cdots & x_{2, j} & \cdots & x_{2, j \prime} & \cdots & x_{2, m} \\
\vdots & \ddots & \vdots & \ddots & \vdots & \ddots & \vdots \\
0 & \cdots & 1 & \cdots & 0 & \cdots & 0 \\
\vdots & \ddots & \vdots & \ddots & \vdots & \ddots & \vdots \\
x_{n, 1} & \cdots & x_{n, j} & \cdots & x_{n, j} & \cdots & x_{n, m}
\end{array}\right)
$$

$X$ being a feasible solution, constraints (7) and (11) of the optimization problem hold: all variables $x_{i j}$ in a line are equal to 0 except one equal to 1 , and $x_{i j}=0$ if $r_{i j}=0$.

The neighborhood $V(X)$ of a feasible solution $X$ is the set $V(X)=$ $\left\{X^{1}, X^{2}, \ldots, X^{e}, \ldots, X^{L(X)}\right\}$. It is composed of all the feasible solutions where 
only a single station has changed of access point compared to the solution $X$. Basically, a neighbor $X^{e}$ is then equal to $X$ except that two elements have been permuted in a same line. These two elements are chosen in such a way that they respect the constraints. Formally, let $X=\left(x_{i j}\right)_{i, j}$ be a feasible solution. A $n \times m$ matrix $X^{e}=\left(x_{i j}^{e}\right)_{i, j}$ belongs to $V(X)$ if and only if $\exists(l, p) \in\{1, . ., m\}^{2}, l \neq p$, and $k \in\{1, . ., n\}$ such that:

- $x_{k l}=x_{k p}^{e}=1$,

- $x_{k p}=x_{k l}^{e}=0$,

- $r_{k p}>0$,

- $x_{i j}=x_{i j}^{e} \forall(i, j) \neq(k, p), \forall(i, j) \neq(k, l)$.

For instance, a neighbor of the matrix $X$ (given above) can be:

$$
X^{e}=\left(\begin{array}{ccccccc}
x_{1,1} & \cdots & x_{1, j} & \cdots & x_{1, j \prime} & \cdots & x_{1, m} \\
x_{2,1} & \cdots & x_{2, j} & \cdots & x_{2, j \prime} & \cdots & x_{2, m} \\
\vdots & \ddots & \vdots & \ddots & \vdots & \ddots & \vdots \\
0 & \cdots & 0 & \cdots & 1 & \cdots & 0 \\
\vdots & \ddots & \vdots & \ddots & \vdots & \ddots & \vdots \\
x_{n, 1} & \cdots & x_{n, j} & \cdots & x_{n, j} & \cdots & x_{n, m}
\end{array}\right)
$$

The neighborhood of a solution $X$ contains at most $(n \times m)-1$ elements $(L(X) \leq n \times m-1$ for all $X)$. The cardinality $L($.$) may be less than$ $(n \times m)-1$ as only feasible solutions are considered: we can move a station $k$ from $A P_{l}$ to $A P_{p}$ only if $r_{k p}>0$.

\subsubsection{Local search algorithm}

The iterative local search algorithm is given in Algorithm 1. It starts from an initial feasible solution $X^{0}$. At each iteration, it computes the objective function for all the neighbors of the current solution. After an iteration, there is at most one station that changes of AP. It corresponds to the best neighbor of the current solution, i.e. the one that maximizes the objective function.

The condition to exit the optimization loop is implemented by the function Convergence() which returns a boolean. This function is not fixed and depends on the context. For instance, Convergence() may return TRUE when it has: 


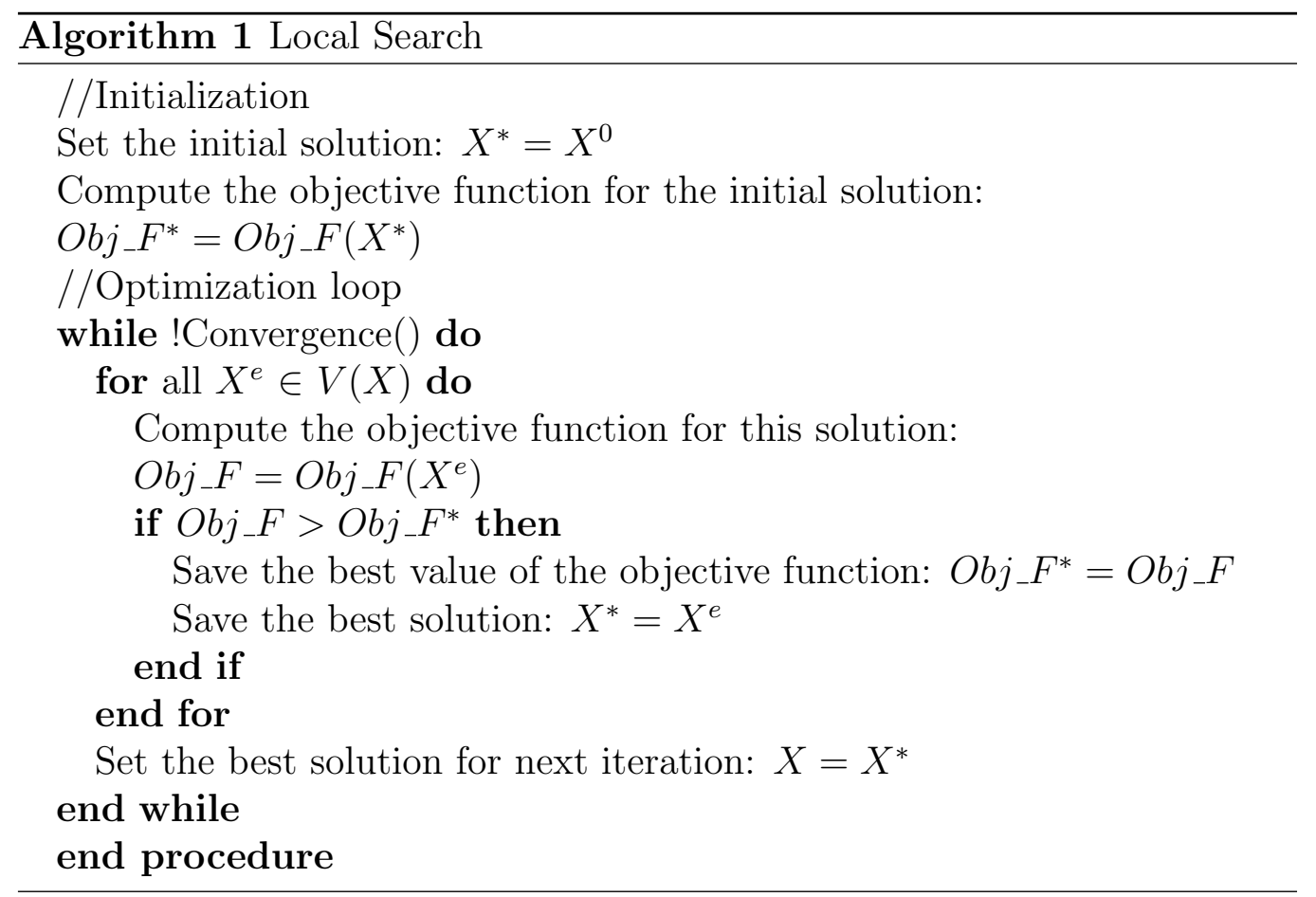

1. found a local maximum (as the solution space is finite, the local search reaches a local optima in a finite number of iterations when the current solution has no neighbor with a greater objective function),

2. reached a maximum fixed threshold for the number of iterations,

3. or exceeded a maximum fixed threshold for the runtime of the optimization program.

The two last conditions ensure that a feasible and better solution (compared to the initial one) may be found while respecting the time constraint of the system.

The result of the local search algorithm greatly depends on the initial solution (i.e. the starting point of the search algorithm $X^{0}$ ). Some of them give a globally optimal solution while others give only locally optimal solutions. Also, the number of iterations to reach the output solution depends on the initial configuration. A way to improve the performance of the algorithm consists in running several instances with different starting points. In this case, Algorithm 1 is repeated several times, with, each time, a different starting solution. This aspect is further discussed in Section 3.3 . 


\subsection{Heuristic evaluation}

In order to assess the effectiveness of the proposed heuristic for solving the optimization problem, we perform tests over 100 different network configurations. We consider 4 access points deploy as a grid. We assign to each AP an orthogonal channel. 20 stations are then deployed around these APs. An example of such a topology is shown in Figure 3 (but with 25 APs and 100 stations in this example). The configurations are obtained by randomly changing stations and APs location. In this part, we use the network simulator ns-3 [38] to generate topologies and extract the initial configurations which are based on RSSI values, whereas the main features of our heuristic are evaluated with the $\mathrm{C}++$ code implementing this heuristic. The capacities $r_{i j}$ are assumed to be known (we explain in the next section how to get them).

\begin{tabular}{|c|c|c|c|c|c|c|c|c|c|}
\hline \multirow[t]{2}{*}{ Conf } & RSSI & \multicolumn{2}{|c|}{ Brute Force } & \multicolumn{3}{|c|}{ Local Search (RSSI) } & \multicolumn{3}{|c|}{ Local Search (multi-start) } \\
\hline & Obj. F & Obj. F & Time(s) & Obj. F & Iter & Time $(\mathrm{ms})$ & Obj. F & Iter & Time $(\mathrm{ms})$ \\
\hline 1 & 17.661 & 21.474 & 225.16 & 21.474 & 6 & 0.233 & 21.474 & 359.00 & 9.943 \\
\hline 2 & 18.604 & 21.038 & 57.24 & 21.038 & 6 & 0.185 & 21.038 & 355.00 & 9.328 \\
\hline 3 & 18.320 & 19.487 & 1800.56 & 19.487 & 4 & 0.149 & 19.487 & 452.00 & 14.394 \\
\hline 4 & 18.451 & 20.896 & 12806.50 & 20.896 & 5 & 0.202 & 20.896 & 406.00 & 13.286 \\
\hline $\mathbf{5}$ & 19.018 & 21.226 & 399.98 & 21.226 & 5 & 0.154 & 21.226 & 386.00 & 10.850 \\
\hline 6 & 19.275 & 21.364 & 712.93 & 21.364 & 6 & 0.196 & 21.364 & 408.00 & 12.164 \\
\hline 7 & 20.034 & 23.442 & 2381.80 & 23.442 & 6 & 0.207 & 23.442 & 400.00 & 12.429 \\
\hline 8 & 17.807 & 21.417 & 2436.58 & 21.417 & 11 & 0.338 & 21.417 & 426.00 & 13.447 \\
\hline 9 & 20.367 & 22.403 & 15090.80 & 22.392 & 4 & 0.165 & 22.403 & 418.00 & 14.118 \\
\hline 10 & 18.896 & 20.832 & 46.86 & 20.832 & 5 & 0.152 & 20.832 & 368.00 & 9.556 \\
\hline 100 & 19.154 & 22.016 & 3028.12 & 22.016 & 4 & 0.142 & 22.016 & 435.00 & 13.509 \\
\hline Mean & 19.008 & 21.402 & 2729.568 & 21.396 & 5.57 & 0.183 & 21.402 & 391.06 & 11.724 \\
\hline
\end{tabular}

Table 3: Comparison results between the Local Search Algorithm and Brute Force Algorithm

For each network configuration, we consider different initial associations (i.e. initial feasible solutions). The first one is based on the RSSI (each station is associated with the AP that gives the best signal strength). We also test the local search algorithm in multi-start mode with 30 different initial associations. For each network configuration, we keep, among the 30 initial instances randomly chosen among the feasible solutions, the one that gives, after optimization, the best result with regard to the objective function. We give, in Table 3, the values of the objective function before optimization ("RSSI" column) and after optimization with the initial association based on RSSI ("Local search (RSSI)" column) and based on 30 initial associations ("Local search (multi-start mode)" column). Beside, we also consider the optimum solution: for a given network configuration, we evaluate the objective 
function for all possible associations and keep the best one ("Brute force" column).

The first observation is that the default association based on RSSI is not optimal. The objective function at the optimum is increased by $10-20 \%$ compared to the RSSI based association. With our heuristic in multi-start mode, the local search algorithm is able to find the optimum in all cases, i.e. for the 100 network configurations. When our heuristic starts from the RSSI association, the optimum is found for 87 configurations over 100. For the other 13 configurations, the maximum difference is less than $1 \%$.

Also, we have evaluated, for each algorithm, the number of iterations and computation times to converge towards its solution. Computation times have been measured on a laptop (RAM 8GB, CPU Core i7 " $4 \times 1.8 G H z "$, OS Linux - Ubuntu). The number of iterations and computation times of the brute force algorithm vary according to the network configurations. It is due to the constraints of our optimization problems, more precisely to the number of APs in the communication range of each station, which leads to a different number of feasible solutions. The optimum is obtained after 8.49 seconds in the best case and after approximately 6 hours (21368 seconds) in the worst case. It is approximately $15 \times 10^{6}$ times greater than for the local search algorithm. It empirically proves that an exhaustive search is not an accurate approach in terms of complexity even for such simple configurations. The multi-start mode of our algorithm requires between 300 and 456 iterations to find its solution (we sum the number of iterations over the 30 instances), corresponding to $7.535 \mathrm{~ms}$ and $15.294 \mathrm{~ms}$ respectively. The local search algorithm based on RSSI needs only 11 iterations in the worst case, with 5.57 iterations in average. The time to obtain the optimum or local optimum takes $0.184 \mathrm{~ms}$ in average, with a maximum of $0.338 \mathrm{~ms}$. This variant of our algorithm is clearly more efficient than the other ones. The number of iterations is at least two times less than with the multi-start mode (13.03 iterations for an instance of the multi-start mode in average versus 5.57 for the one based on RSSI).

It clearly appears that the heuristic starting from the default RSSI association offers an interesting tradeoff between performance and complexity. It leads to an efficient solution, close to the optimum, and requires only one initial configuration. This initial configuration seems relevant as it converges faster to the solution compared to a random initial configuration. Consequently, it is this variant of our algorithm that will be used in the performance evaluation part presented in the next section. 


\section{Performance Evaluation}

We now use the network simulator ns-3 to evaluate the performance of our heuristic with a more realistic and richer environment, as all network aspects from the physical to the application layers are simulated.

Simulations are performed as follows. The first step consists in using ns-3 to create the network topologies, to compute the link capacities between the APs and the stations $\left(r_{i j}\right)$ and to extract the initial association based on the RSSI values. A link capacity between one AP and one station corresponds to the throughput received by the station when a saturated constant bit rate (CBR) flow is generated between the two considered nodes and when all other stations and interferences from the other $\mathrm{AP} /$ stations are neglected. Note that these capacities are computed at the application layer of the TCP/IP stack. This has the advantage of: i) taking into account the headers generated by the sub-layers and the overhead induced by the IEEE 802.11 DCF mode

(e.g. the MAC header and the Acknowledgment frame), ii) directly obtaining the useful throughput, iii) designing the proposed model independently of the standard $(802.11 \mathrm{a} / \mathrm{b} / \mathrm{g} / \mathrm{n}, \ldots)$.

In a second step, ns-3 generates CBR traffic between APs and stations for the RSSI association. This traffic is homogeneous between stations and saturates the medium. The generated payloads have a size of 1500 bytes. We then measure the obtained throughput $\left(d_{i j}\right)$ for each station.

The last step consists in running our heuristic to find an optimized association. This step has been integrated to ns-3. Once our heuristic has found the solution, we force the stations to associate to the corresponding APs. We then generate again the same CBR traffic between APs and stations and measure again the obtained throughput $\left(d_{i j}\right)$ for each station. Other types of traffic are also tested. They are described when they are specifically tested.

As the objective function aims to offer a trade-off between throughput and fairness, the performance metrics we consider are the overall throughput (sum of the throughput for all stations) and the Jain's index. These two metrics are computed from the ns-3 simulations.

The Jain's Index [39] evaluates the fairness achieved in the network. It is defined as follows: 


$$
\text { Jain }=\frac{\left(\sum_{i=1}^{n} d_{i A P(i)}\right)^{2}}{n \sum_{i=1}^{n} d_{i A P(i)}^{2}}
$$

where station $i$ is associated with $\operatorname{AP}(\mathrm{i})$.

Wireless interfaces are configured to use the IEEE $802.11 \mathrm{n}$ standard. We shall simulate it on the two frequency bands: $2.4 \mathrm{GHz}$ and $5 \mathrm{GHz}$. The transmission power is $40 \mathrm{~mW}(16.00206 \mathrm{dBm})$. We use the rate adaptation algorithm IdealHtWifiManager of ns-3 to set the physical rate between stations and APs. We had to develop it as it was not available for 802.11n. The code may be found in [40]. This manager determines the best physical transmission rate to use between a station and its AP according to the SNR measured on packets sent from the source to the destination.

The Wi-Fi network consists of $25(5 \times 5)$ access points, deployed on a square grid such that the distance between two adjacent APs is 100 meters. APs are then randomly moved within a circle with a diameter of 25 meters (the center being the grid points) to obtain more realistic topologies. This distance leads to overlapping zones. A station may then have several choices for its association. Stations are randomly distributed in the coverage area of the access points. The distribution is Gaussian, centered in the middle of the grid. A topology sample is shown in Figure 3.

For each scenario, the number of APs is fixed (25), and we increase the number of wireless stations from 25 to 250 . For each scenario, we perform 30 different configurations for a given number of stations. These configurations are obtained by randomly changing the station and AP positions. In the different figures, each point is the mean of these 30 simulations with a confidence interval at $95 \%$.

\subsection{Orthogonal channels}

Figure 4 illustrates the performance results when all APs have orthogonal channels in the $2.4 \mathrm{GHz}$ band. This scenario enables to show the solution performance when there is no radio conflict. Figure 4 a represents the overall network throughput when associations are based on RSSI values and our heuristic (based on the first model (Eq. 7)).

We observe that our algorithm improves the overall throughput by about $40 \%$ for a low number of stations, and by $20 \%$ when the number of stations 


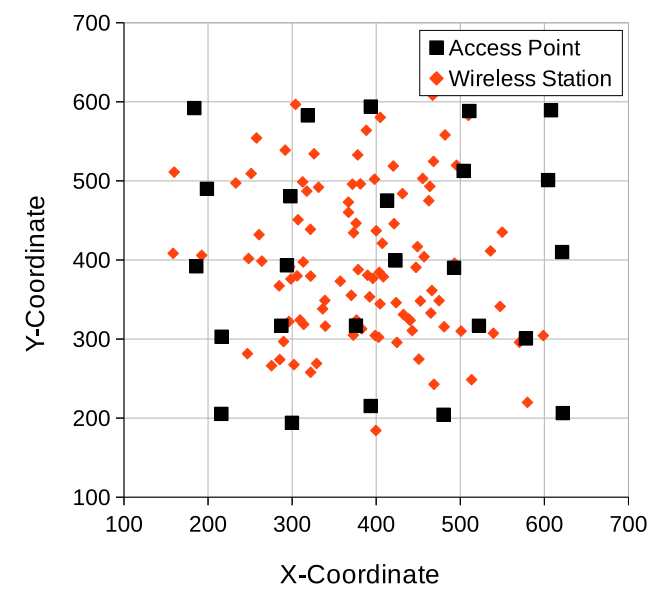

Figure 3: Placement of APs and wireless stations for one simulation.

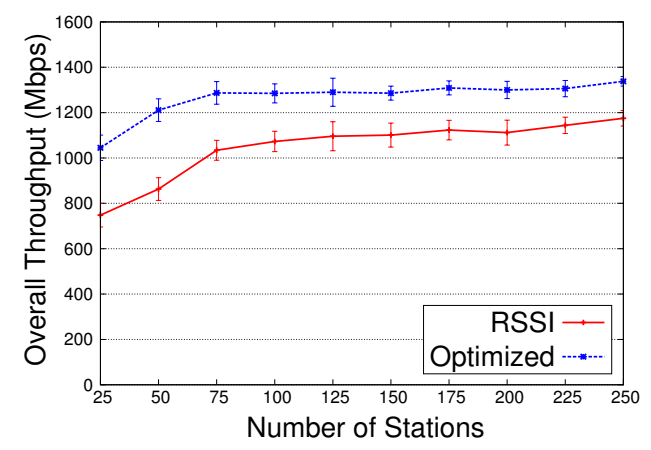

(a) Overall Network Throughput.

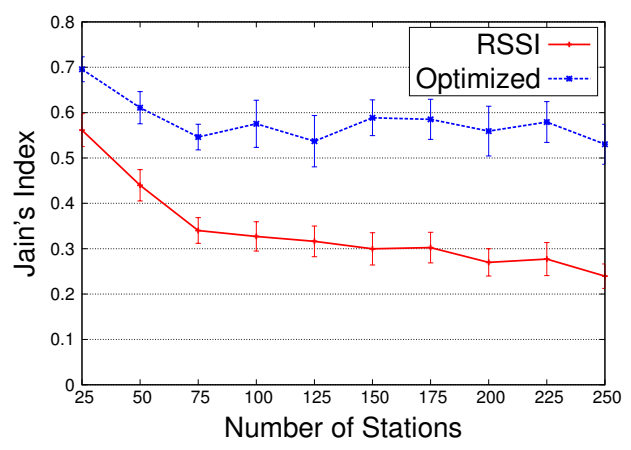

(b) Jain's Fairness Index.

Figure 4: All Orthogonal channels.

reaches 250. Also, we can see that the overall throughput of the network increases until 75 stations ( 3 stations per access point in average) and remains stable for a greater number of stations. Figure $4 \mathrm{~b}$ shows the evolution of the Jain's Fairness index before and after optimization. The optimization significantly improves the fairness, up to $120 \%$ for 250 stations. Moreover, we observe that, with the RSSI association, the fairness decreases with the number of stations whereas it seems to remain stable with our algorithm (at least with 75 stations and more).

Fairness is also illustrated in Figure 5, where for one simulation (250 stations), we plot the distribution of the number of stations associated to 


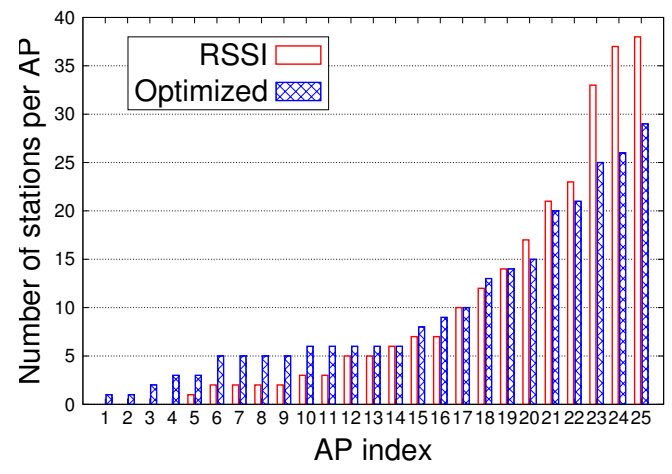

(a) Number of stations per AP.

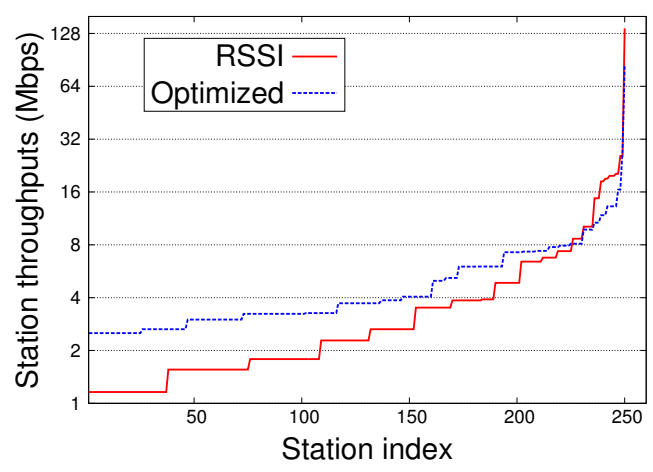

(b) Throughput per Station.

Figure 5: Comparison of the number of stations per AP and station throughput for one simulation sample before (RSSI) and after optimization (Optimized).

each AP, and the station throughput $\left(d_{i j}\right)$ before and after optimization. This simulation is representative: the observed trends are similar for all simulations. In Figure $5 \mathrm{a}$, we can observe that 4 APs do not have any stations associated with them with the RSSI association, whereas there is only one AP without station after optimization. With our solution, it appears that stations are more homogeneously distributed between APs compared to the RSSI case. A more homogeneous distribution of stations among the APs leads to more balanced throughput among stations (Figure 5b). In this figure, the $\mathrm{x}$-axis represents the indexes of the 250 stations in an increasing order of the station throughput. The y-axis represents the station throughput (with a $\log$ scale). It varies from $1.15 \mathrm{Mb} / \mathrm{s}$ to $136 \mathrm{Mb} / \mathrm{s}$ for the RSSI association, and from $2.51 \mathrm{Mb} / \mathrm{s}$ to $83 \mathrm{Mb} / \mathrm{s}$ after optimization. It clearly shows a better usage of Wi-Fi resources: stations use more APs and they are more homogeneously shared between APs leading to a better fairness and a throughput increase.

\subsection{Non-orthogonal channels}

We simulate two cases: one in the $5 \mathrm{GHz}$ band with 8 orthogonal channels and one in the $2.4 \mathrm{GHz}$ band with 3 orthogonal channels. We distribute channels on APs in a way that minimizes the number of conflicts and interference. It corresponds to a scenario where the AP deployment has been planned. Figure 6a (8 orthogonal channels) shows that our optimization (based on the second model (Eq. 11)) improves the overall throughput up to $20 \%$ regardless of the number of stations. The Jain's Fairness index, shown 


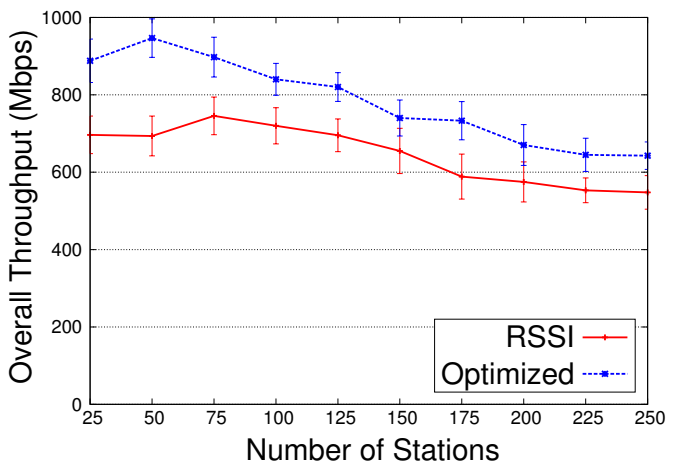

(a) Overall Network Throughput.

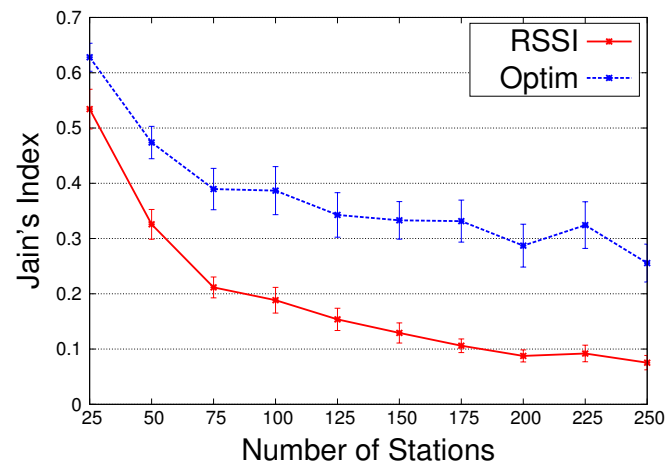

(b) Jain's Fairness Index.

Figure 6: 8 Orthogonal channels.

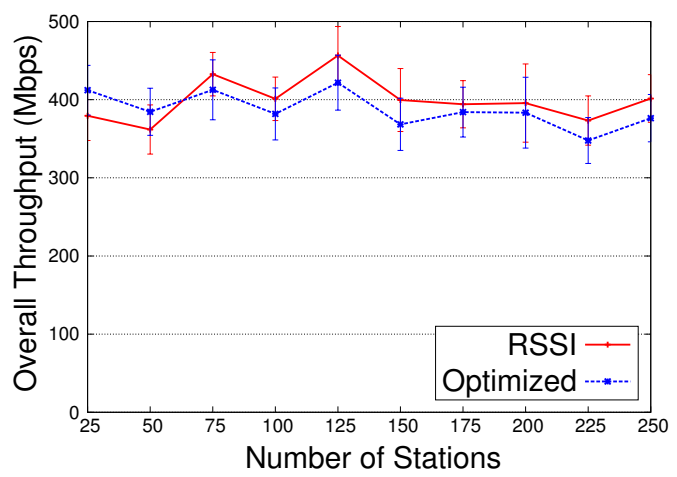

(a) Overall Network Throughput.

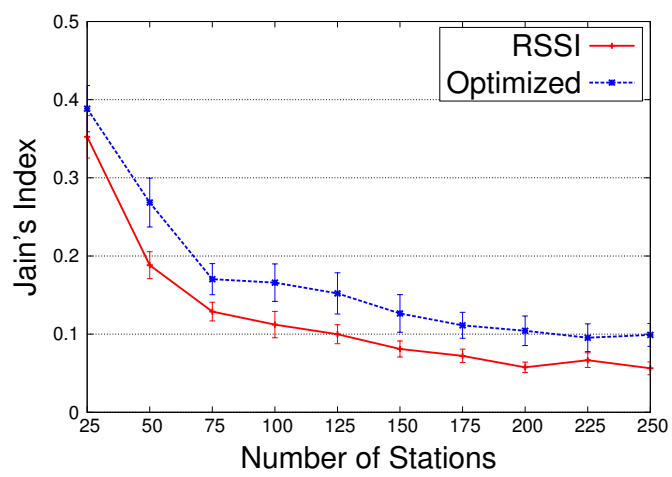

(b) Jain's Fairness Index.

Figure 7: 3 Orthogonal channels.

in Figure 6b, is improved by our optimization by a factor varying from 1.18 for 25 stations to 3.39 for 250 stations.

Figure 7 illustrates the simulation results obtained in the $2.4 \mathrm{GHz}$ band with 3 orthogonal channels. Improvements are clearly less significant than in the 8 channels case. Figure $7 \mathrm{a}$ shows that the optimization does not increase the overall throughput and the obtained results are almost equivalent with these two solutions, with a small advantage to the RSSI-based association. Nevertheless, we can observe an improvement of the Jain's index, with our optimization, varying from $10 \%$ to $100 \%$ (35\% in average).

In our simulations, the sensing range is approximately 221 meters. With our channel allocation and our topologies, an AP detects transmissions from 
at most 3 APs. As we have seen during the formulation of the problem, APs/stations that share the medium tends to obtain the same throughput. Consequently, in this very constrained scenario, performance can not be significantly improved. Throughput can hardly be increased since the high number of stations on each channel does not allow to separate stations with high and low link capacities, and fairness is already imposed by stations with low link capacities.

Variable packet sizes. To assess the impact of the packet size, that we have assumed the same for all stations in the proposed model, we simulate the same scenario with packet sizes that vary according to a distribution designed from a real trace [41]. The packet size distribution of this trace is shown in Figure 8. The sent packets have then different size but the average packet size is the same as in the previous simulations (Average Packet Size $=755.572$ bytes and Standard Deviation $=674.05)$. The simulation results, for the case of 8 orthogonal channels, are illustrated in Figure 9, In Figure 9a, we show that the overall throughput is improved in average of about $20 \%$ with a peak of $36 \%$ at 50 stations with our optimization. Jain's index is plotted in Figure $9 \mathrm{~b}$ where we observe an improvement of $90 \%$ in average. We also used this trace to simulate flows with different average packet sizes. The mean, standard deviation, minimum value and the maximum value of the packet sizes sent by each station are summarized in Table 4. The simulation results are illustrated in Figure 10. Figure 10a shows that the overall throughput is improved of $22 \%$ in average after the association optimization. Figure $10 \mathrm{~b}$ shows that fairness is improved of $88 \%$ in average.

\begin{tabular}{|c|c|c|}
\hline \# of stations & $\mathbf{2 5}$ & $\mathbf{2 5 0}$ \\
\hline \hline Mean & 887.42 & 864.75 \\
StDev & 149.05 & 155.17 \\
Min & 594.96 & 396.14 \\
Max & 1088.12 & 1186.38 \\
\hline
\end{tabular}

Table 4: Size properties of the transmitted packets in case of variable packet sizes

Non saturated networks. We tested a scenario where the network is not saturated. We consider satisfied and non-satisfied stations. A station is satisfied when its throughput corresponds to its demand (the one generated by its application in our simulations). Then, to evaluate the impact of the association 


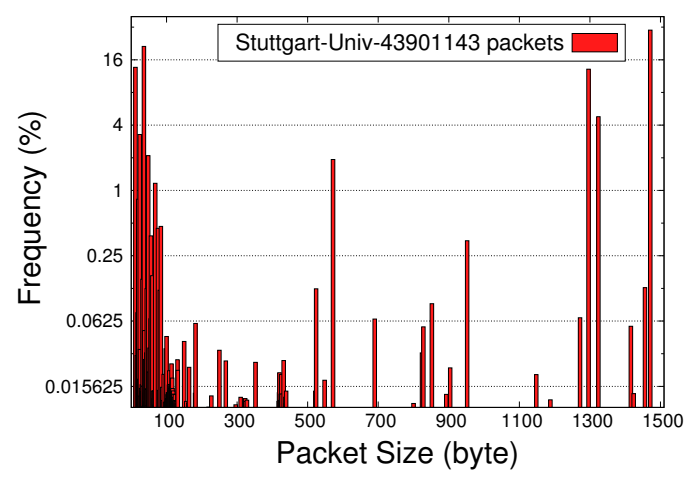

Figure 8: Packet size histogram from a trace of the University of Stuttgart.

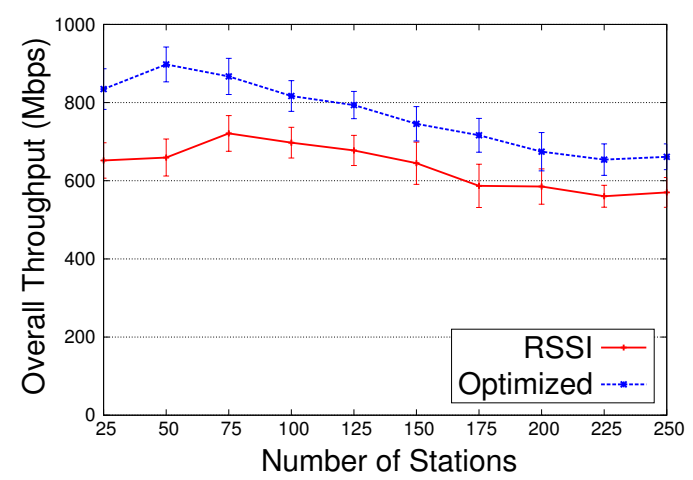

(a) Overall Network Throughput.

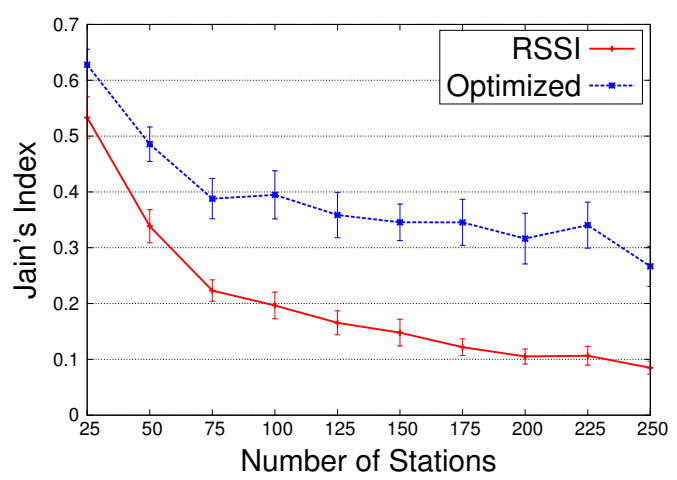

(b) Jain's Fairness Index.

Figure 9: Packets with different sizes but with an identical mean for each station (8 orthogonal channels)

optimization on the satisfaction of each station, we count the number of satisfied stations in the network before and after optimization. In this scenario we have kept the same topology with a network of 100 stations and 25 APs configured on 8 orthogonal channels. The results are shown in Figure11. For RSSI association (first bar) we have in average 25 stations satisfied whereas we have more than 42 stations satisfied after optimization. It represents of number of satisfied stations of $73 \%$. The three other bars represent the number of stations changing from a state to an other after optimization (for instance "0-1": from unsatisfied to satisfied). We observe that $27.33 \%$ of stations have changed from unsatisfied to satisfied state after optimization ("0-1") and only $9.53 \%$ have changed from satisfied to unsatisfied ("1-0"). 


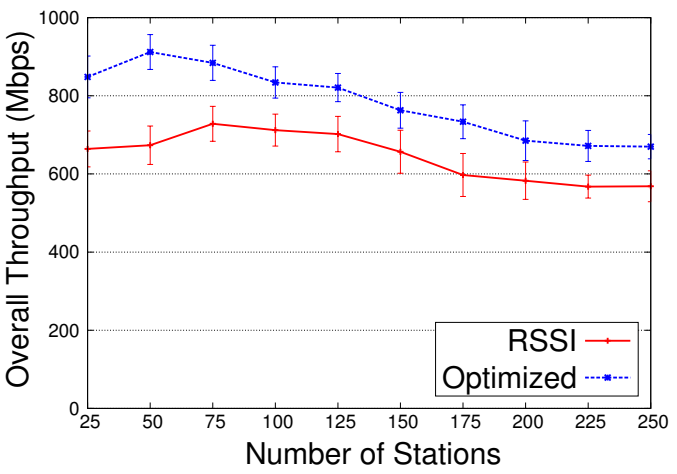

(a) Overall Network Throughput.

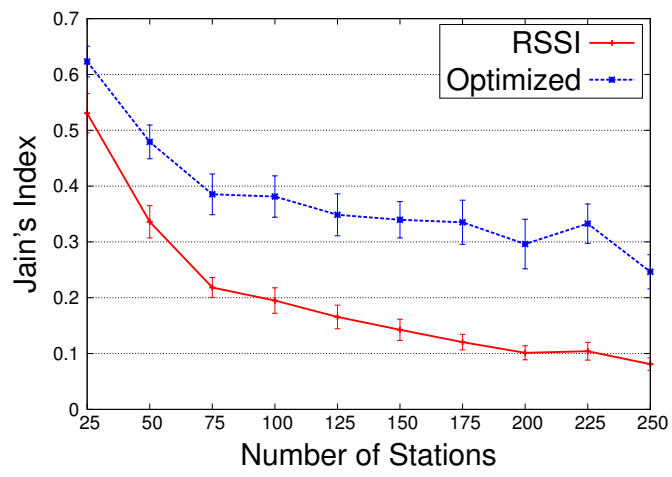

(b) Jain's Fairness Index.

Figure 10: Packets with a different average size for each station (8 orthogonal channels)

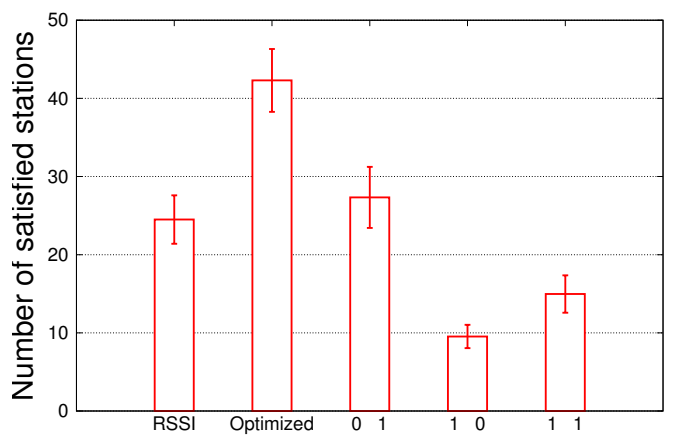

Figure 11: Number of satisfied stations before and after optimization.

\subsection{On-Line Optimization}

Our optimization can be used, in practice, in an on-line way. More precisely, it may be run at regular interval or trigger when an event occurs, to take into account stations that have left or joined the Wi-Fi network or that have moved. To illustrate the dynamic behavior of our approach, we simulated on the same network topology another scenario in which we randomly remove 50 stations among the 250 and we replace it with 50 new ones at each interval. The new locations follow the same distribution as the initial one. The new stations first associate to APs according to the RSSI value, and then our optimization is applied. We repeat this scenario 10 times. The results with 8 orthogonal channels are shown in Figure 12. The "Non optimized" evaluation corresponds to a configuration where the association of 


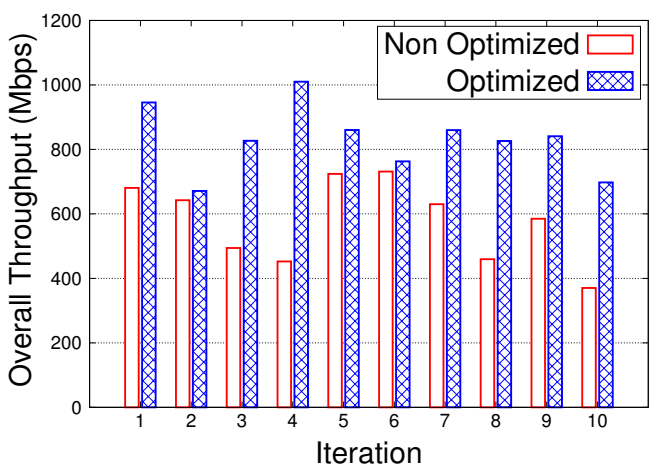

(a) Overall Throughput.

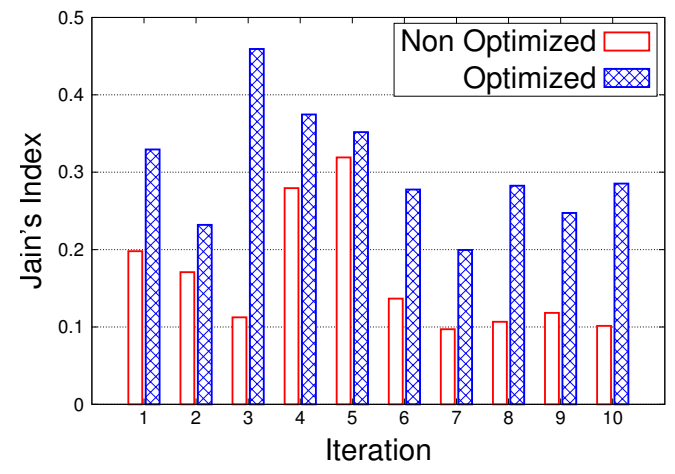

(b) Jain's Fairness Index.

Figure 12: Overall throughput and Jain's index for dynamic association optimization

the 200 stations that remain come from the previous optimization and the 50 new stations are associated in function of the RSSI. Figure 12a shows the improvement of the overall network throughput after the optimization at each iteration. This improvement varies between $5 \%$ and $120 \%$. Figure $12 \mathrm{~b}$ shows an average fairness improvement of $110 \%$ by optimization.

From these results, we note that even if only $20 \%$ of the stations change of position in an already optimized configuration, our solution still allows a significant improvement of the overall network performance.

\subsection{TCP traffic}

We also tested our solution with TCP traffic. The scenario is the same as the one evaluated with CBR traffic, except that traffic is replaced by TCP flows. The use of TCP allows us to test the robustness of our model with respect to TCP when stations are in competition for accessing the medium for the TCP acknowledgment transmissions. In Figure 13 , we can observe that the results are qualitatively the same: there is an increase of $20-30 \%$ of throughput, and $20-90 \%$ for fairness. It empirically shows that the different TCP mechanisms (congestion/flow control) and the upload traffic generated by the TCP acknowledgments do not affect the accuracy of our model.

\section{Comparison with time-based approach and discussion}

In this section, we compare the results, obtained with ns-3, given by our optimization model which assumes an access-based fairness (named AbF 


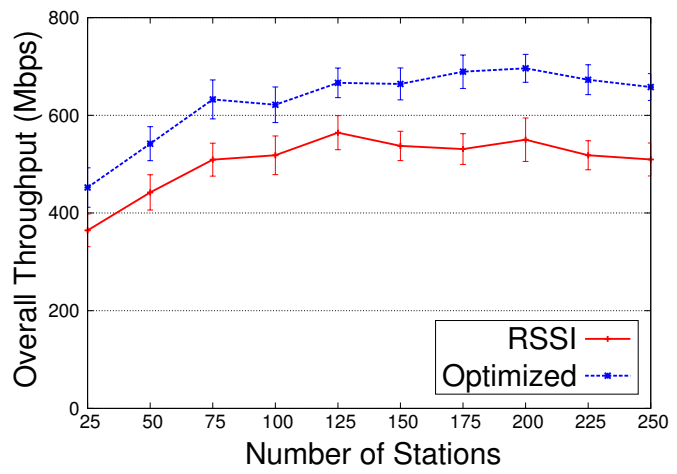

(a) Overall Network Throughput.

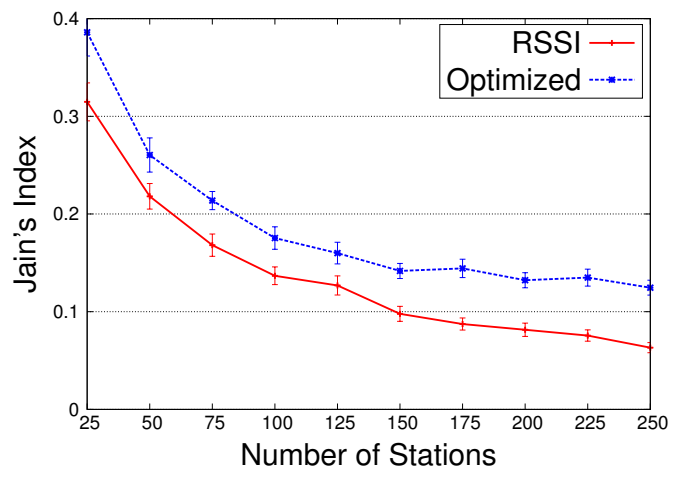

(b) Jain's Fairness Index.

Figure 13: 8 Orthogonal Channels with TCP flows

hereafter) with the model proposed in [15, 16, 17, 19] that is based on a time-based fairness (named TbF hereafter). We remind that in the TbF model, the medium is equally shared in time between the stations associated to a same AP while the medium is equally shared in access between APs in conflict. We made the required modification in ns-3 to respect this model.

Figures 14 and 15 present the results obtained by the two models in case of 8 orthogonal and 3 orthogonal channels respectively. We observe almost the same overall throughput for 8 orthogonal channels (Figure 14a). For 3 orthogonal channels, results are equivalent until 75 stations but the difference can reach up to $30 \%$ for 250 stations in favor of the AbF algorithm (Figure 15a). Jain's index is almost equivalent whatever the number of orthogonal channels.

These results are surprising, because, intuitively, the TbF model should lead to a better throughput in all cases since, with the TbF model, stations with high physical rates are not penalized by the presence of low physical rate stations (unlike the AbF model). That may be explained by the increase of the number of APs in conflict with the decrease of the number of orthogonal channels. Indeed, with 3 orthogonal channels, each AP is very likely in conflict with at least one another AP and in this configuration, the gain expected with the use of TbF model is reduced.

We simulate a network of 10 APs and 100 stations with AbF and TbF schedulers to understand how our optimization algorithm allocates the stations according to their physical rate. So as to take into account the physical rate of stations regardless of their positions relative to the APs, we install 


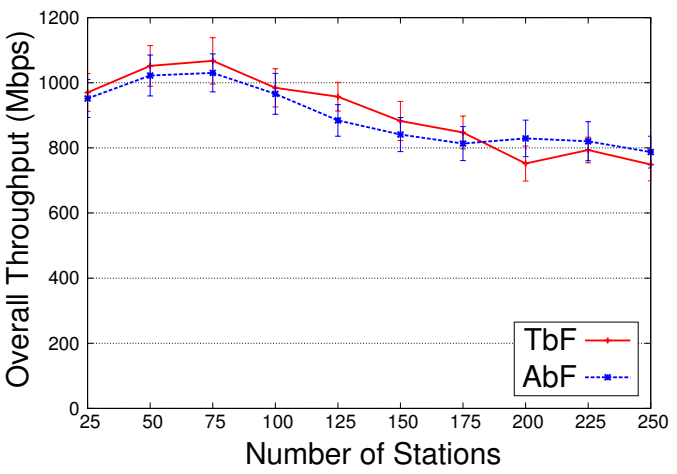

(a) Overall Network Throughput.

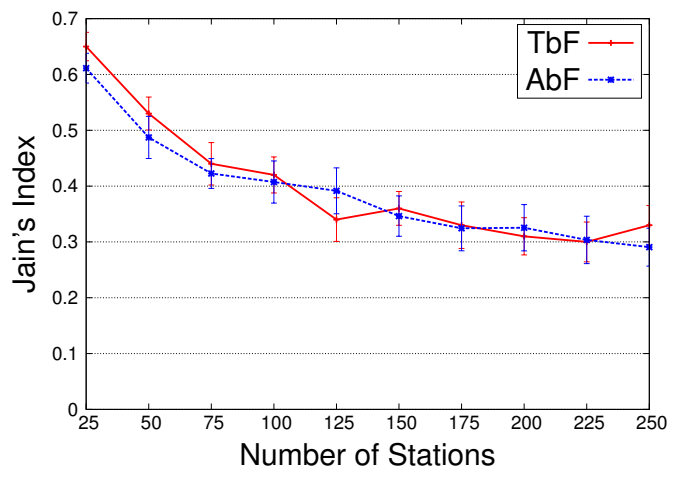

(b) Jain's Fairness Index.

Figure 14: 8 Orthogonal Channels

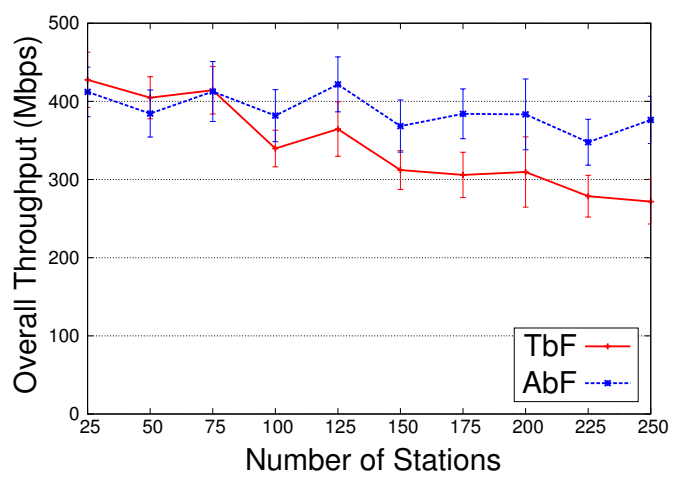

(a) Overall Network Throughput.

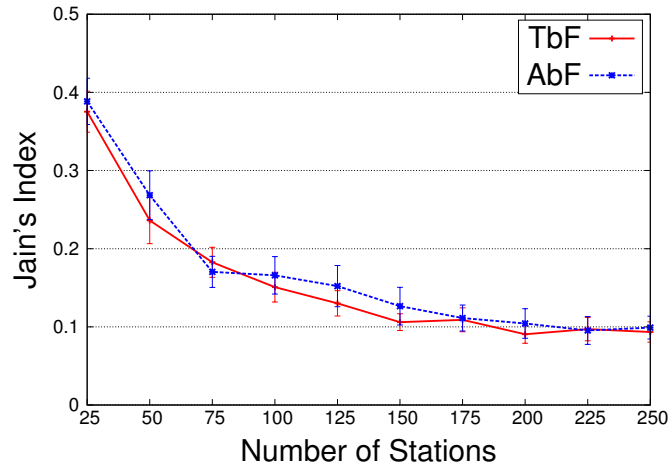

(b) Jain's Fairness Index.

Figure 15: 3 Orthogonal Channels

all the APs at the same location and the stations are uniformly distributed around. Simulation results plotted in Figure 16 show the distribution of stations on the APs ordered by their physical rates.

For the case of AbF (Figure 16a), it appears that our algorithm tends to associate stations with the same physical rates to the same AP. Stations with high physical rates are consequently not penalized by low rates stations and the global throughput is increased. Also, to ensure fairness, it fairly distributes stations between the 8 orthogonal channels. Instead, in the case of $\mathrm{TbF}$ (Figure 16b), we notice that the stations are distributed homogeneously between APs so they have the same number of stations with high and low physical rates. 


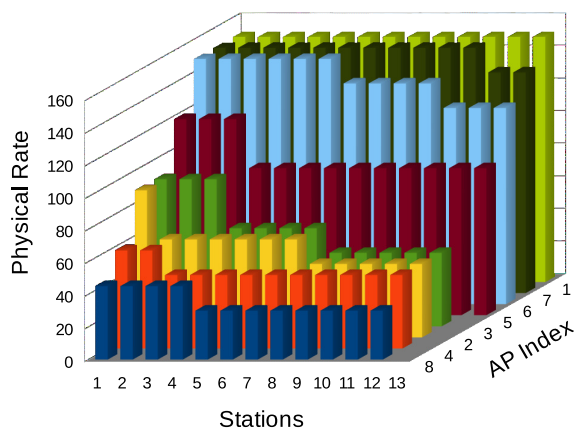

(a) $\mathrm{AbF}$.

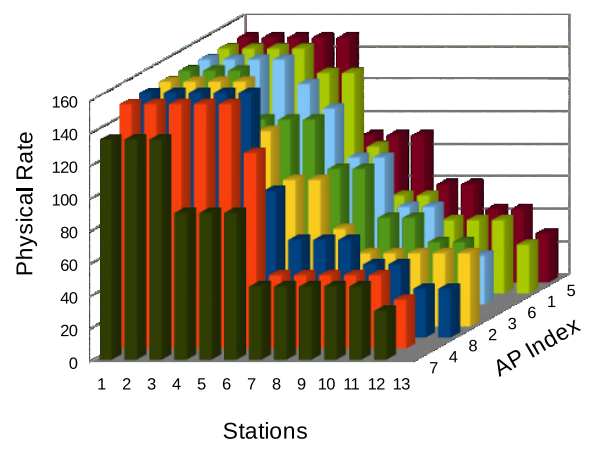

(b) $\mathrm{TbF}$.

Figure 16: Per-AP station physical rate for $\mathrm{AbF}$ and $\mathrm{TbF}$ (8 orthogonal channels).

\section{Conclusion}

In this paper, we address the association problem in Wi-Fi networks. Our solution, based on an optimization model, aims to improve the overall network throughput while achieving a better fairness between stations, compared to the classical association based on RSSI.

Our proposed optimization solution is based on a mathematical formulation of the problem and a local search algorithm. The benefit of this algorithm is a convergence in a few iterations when the starting point is the default RSSI association. Moreover, the algorithm can be stopped at any time and always gives a feasible and better association. It can be easily tuned according to the CPU or time constraints of the WLAN controller. Simulation results show that the proposed optimization significantly improves the network performance. In case of orthogonal channels, our optimization increases the overall throughput up to $40 \%$ and the fairness up to $120 \%$, and for non-orthogonal channels we observe an improvement varying from $15 \%$ to $40 \%$ for the overall throughput and from $25 \%$ to $300 \%$ for the fairness. This improvement is due to a better distribution of stations among the AP, and an improvement throughput for most of the stations.

Our solution has also been validated when TCP traffic are transmitted and for different types of traffic (packet sizes, saturated and non saturated). Moreover, we have shown that the use of an access-based fairness scheme, in addition to be more realistic, leads to better results when the number of orthogonal channels is limited compared to the use of a time-based fairness scheme. 
This work could be extended by taking into account the structure of the neighborhood in the local search algorithm to improve the efficiency of our algorithm. For instance, we could consider only a sub-set of neighbors which (a priori) increases the objective function. It could also be interesting to include the users' traffic demand in our model. Rather than providing fairness to users independently of their current load, it would lead to associations that allocate resources to stations that need them for a given period.

\section{References}

[1] Cisco visual networking index: Forecast and methodology, 2016-2021, White Paper.

[2] IEEE standard for information technology-telecommunications and information exchange between systems local and metropolitan area networks-specific requirements part 11: Wireless LAN medium access control (MAC) and physical layer (PHY) specifications, IEEE Std 802.11-2012 (2012) 1-2793.

[3] IEEE Std 802.11n-2009 (Amendment to IEEE Std 802.11-2007 as amended by IEEE Std 802.11k-2008, IEEE Std 802.11r-2008, IEEE Std 802.11y-2008, and IEEE Std 802.11w-2009) (2009) 1-565.

[4] IEEE Std 802.11ac-2013 (Amendment to IEEE Std 802.11-2012, as amended by IEEE Std 802.11ae-2012, IEEE Std 802.11aa-2012, and IEEE Std 802.11ad-2012) (2013) 1-425.

[5] L. Zhang, L. Zhao, Z. Wang, J. Liu, Wifi networks in metropolises: From access point and user perspectives, IEEE Communications Magazine 55 (5) (2017) 42-48. doi:10.1109/MCOM.2017.1600262.

[6] A. Farshad, M. K. Marina, F. Garcia, Urban wifi characterization via mobile crowdsensing, in: 2014 IEEE Network Operations and Management Symposium (NOMS), 2014, pp. 1-9. doi:10.1109/NOMS.2014. 6838233 .

[7] P. Calhoun, M. Montemurro, D. Stanley, Control and provisioning of wireless access points (CAPWAP) protocol specification, RFC 5415, RFC Editor (March 2009). 
[8] IEEE Std 802.11v-2011 (Amendment to IEEE Std 802.11-2007 as amended by IEEE Std 802.11k-2008, IEEE Std 802.11r-2008, IEEE Std 802.11y-2008, IEEE Std 802.11w-2009, IEEE Std 802.11n-2009, IEEE Std 802.11p-2010, and IEEE Std 802.11z-2010) (2011) 1-433.

[9] M. Yang, Y. Li, D. Jin, L. Zeng, X. Wu, A. V. Vasilakos, Softwaredefined and virtualized future mobile and wireless networks: A survey, Mobile Networks and Applications 20 (1) (2015) 4-18. doi:10.1007/ s11036-014-0533-8.

[10] M. G. Resende, P. M. Pardalos, Handbook of Optimization in Telecommunication, Springer US, 2006. doi:10.1007/978-0-387-30165-5.

[11] J. Kennington, E. Olinick, D. Rajan, Wireless Network Design: Optimization Models and Solution Procedures, Springer-Verlag New York, 2010. doi:10.1007/978-1-4419-6111-2.

[12] F. D'Andreagiovanni, C. Mannino, A. Sassano, GUB covers and powerindexed formulations for wireless network design, Management Science 59 (1) (2013) 142-156. doi:10.1287/mnsc.1120.1571.

[13] B. Gendron, M. G. Scutellà, R. G. Garroppo, G. Nencioni, L. Tavanti, A branch-and-benders-cut method for nonlinear power design in green wireless local area networks, European Journal of Operational Research 255 (1) (2016) 151 - 162. doi:https://doi.org/10.1016/j.ejor. 2016.04 .058 .

[14] Y. Bejerano, S. J. Han, L. Li, Fairness and load balancing in wireless LANs using association control, IEEE/ACM Transactions on Networking 15 (3) (2007) 560-573. doi:10.1109/TNET.2007.893680.

[15] O. B. Karimi, J. Liu, J. Rexford, Optimal collaborative access point association in wireless networks, in: IEEE INFOCOM 2014 - IEEE Conference on Computer Communications, 2014, pp. 1141-1149. doi: 10.1109/INFOCOM. 2014.6848045.

[16] L. Li, M. Pal, Y. R. Yang, Proportional fairness in multi-rate wireless LANs, in: IEEE INFOCOM 2008 - The 27th Conference on Computer Communications, 2008. doi:10.1109/INFOCOM.2008.154. 
[17] W. Li, S. Wang, Y. Cui, X. Cheng, R. Xin, M. A. Al-Rodhaan, A. AlDhelaan, AP association for proportional fairness in multi-rate WLANs, IEEE/ACM Transactions on Networking 22 (1) (2014) 191-202. doi: 10.1109/TNET.2013.2245145.

[18] M. Collotta, FLBA: A fuzzy algorithm for load balancing in ieee 802.11 networks, Journal of Network and Computer Applications 53 (2015) 183 - 192. doi:https://doi.org/10.1016/j.jnca.2015.04.005.

[19] H. Tang, L. Yang, J. Dong, Z. Ou, Y. Cui, J. Wu, Throughput optimization via association control in wireless LANs, Mobile Networks and Applications 21 (3) (2016) 453-466. doi:10.1007/s11036-015-0650-z.

[20] M. AMER, A. Busson, I. Guérin Lassous, Association optimization in wi-fi networks: Use of an access-based fairness, in: Proceedings of the 19th ACM International Conference on Modeling, Analysis and Simulation of Wireless and Mobile Systems, MSWiM '16, ACM, New York, NY, USA, 2016, pp. 119-126. doi:10.1145/2988287.2989153.

[21] S. Keranidis, T. Korakis, I. Koutsopoulos, L. Tassiulas, Contention and traffic load-aware association in IEEE 802.11 WLANs: Algorithms and implementation, in: 2011 International Symposium of Modeling and Optimization of Mobile, Ad Hoc, and Wireless Networks, 2011, pp. 334341. doi:10.1109/WIOPT.2011.5930036.

[22] H. Gong, J. Kim, Dynamic load balancing through association control of mobile users in wifi networks, IEEE Transactions on Consumer Electronics 54 (2) (2008) 342-348. doi:10.1109/TCE.2008.4560097.

[23] G. Athanasiou, P. C. Weeraddana, C. Fischione, L. Tassiulas, Optimizing client association for load balancing and fairness in millimeterwave wireless networks, IEEE/ACM Transactions on Networking 23 (3) (2015) 836-850. doi:10.1109/TNET . 2014.2307918.

[24] S. K. Dandapat, B. Mitra, R. R. Choudhury, N. Ganguly, Smart association control in wireless mobile environment using max-flow, IEEE Transactions on Network and Service Management 9 (1) (2012) 73-86. doi:10.1109/TNSM. 2011.113011.100098.

[25] W. Wong, A. Thakur, S. H. G. Chan, An approximation algorithm for ap association under user migration cost constraint, in: IEEE INFOCOM 
2016 - The 35th Annual IEEE International Conference on Computer Communications, 2016, pp. 1-9. doi :10.1109/INFOCOM.2016.7524397.

[26] S. Bayhan, A. Zubow, Optimal mapping of stations to access points in enterprise wireless local area networks, in: Proceedings of the 20th ACM International Conference on Modelling, Analysis and Simulation of Wireless and Mobile Systems, MSWiM '17, ACM, New York, NY, USA, 2017, pp. 9-18. doi:10.1145/3127540.3127556.

[27] F. D'Andreagiovanni, R. G. Garroppo, M. G. Scutellà, Power savings with data rate guarantee in dense wlans, in: 2017 International Conference on Selected Topics in Mobile and Wireless Networking (MoWNeT), 2017, pp. 1-8. doi:10.1109/MoWNet.2017.8045946.

[28] F. D'Andreagiovanni, R. G. Garroppo, M. G. Scutellà, Green design of wireless local area networks by multiband robust optimization, Electronic Notes in Discrete Mathematics 64 (2018) 225-234. doi : 10.1016/j.endm.2018.01.024.

[29] J. Hwang, J. Choi, J. Yoo, C.-k. Kim, A theoretical approach to optimal association control in vehicular wi-fi networks, EURASIP Journal on Wireless Communications and Networking 2014 (1) (2014) 192. doi: 10.1186/1687-1499-2014-192.

[30] A. Gupta, J. Min, I. Rhee, WiFox: Scaling Wi-Fi performance for large audience environments, in: Proceedings of the 8th International Conference on Emerging Networking Experiments and Technologies, CoNEXT '12, ACM, New York, NY, USA, 2012, pp. 217-228. doi: 10.1145/2413176.2413202.

[31] F. Malandrino, C. F. Chiasserini, S. Kirkpatrick, Cellular network traces towards 5g: Usage, analysis and generation, IEEE Transactions on Mobile Computing 17 (3) (2018) 529-542. doi:10.1109/TMC.2017. 2737011.

[32] G. Bianchi, Performance analysis of the IEEE 802.11 distributed coordination function, IEEE Journal on Selected Areas in Communications 18 (3) (2000) 535-547. doi:10.1109/49.840210. 
[33] F. P. Kelly, Charging and rate control for elastic traffic, European Transactions on Telecommunications 8 (1997) 33-37. doi:10.1002/ ett. 4460080106 .

[34] G. Berger-Sabbatel, A. Duda, M. Heusse, F. Rousseau, Short-Term fairness of 802.11 networks with several hosts, in: E. M. Belding-Royer, K. Al Agha, G. Pujolle (Eds.), Mobile and Wireless Communication Networks, Springer US, Boston, MA, 2005, pp. 263-274.

[35] T. Bu, L. Li, R. Ramjee, Generalized proportional fair scheduling in third generation wireless data networks, in: Proceedings IEEE INFOCOM 2006. 25TH IEEE International Conference on Computer Communications, 2006, pp. 1-12. doi:10.1109/INFOCOM.2006.145.

[36] D. B. Shmoys, É. Tardos, An approximation algorithm for the generalized assignment problem, Mathematical Programming 62 (1) (1993) 461-474. doi:10.1007/BF01585178.

[37] J. P. Walser, Integer Optimization by Local Search: A Domainindependent Approach, Springer-Verlag Berlin Heidelberg, Berlin, Heidelberg, 1999. doi:10.1007/3-540-48369-1.

[38] NS-3: A discrete-event network simulator for internet systems, version3.23.https://www.nsnam.org/ (2015).

[39] R. Jain, D. M. Chiu, W. R. Hawe, A quantitative measure of fairness and discrimination for resource allocation in shared computer system, Research Report DEC-TR-301, Digital Equipment Corporation (1984).

[40] NS-3 code: Ideal HT Wi-Fi manager, http://perso.ens-lyon.fr/ mohammed.amer/Ideal-ht-wifi-manager.

[41] Institute of Communication Networks and Computer Engineering, Trace of the dormitory network selfnet., http://www.ikr.uni-stuttgart. de/ sass/traces/Welcome.html\#selfnet (2004). 